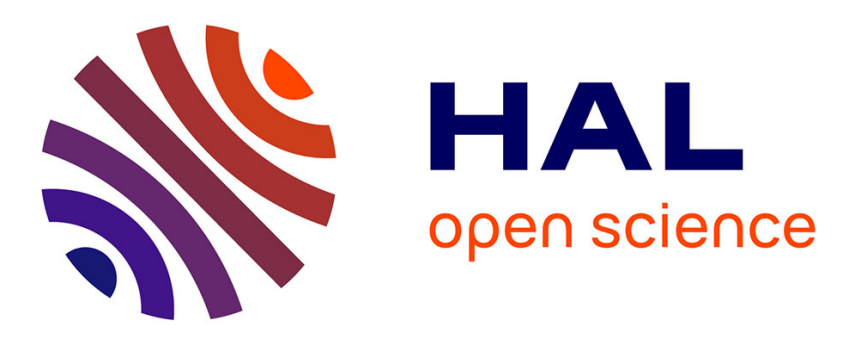

\title{
Models and methods for two-layer shallow water flows
}

\author{
Alexandre Chiapolino, Richard Saurel
}

\section{To cite this version:}

Alexandre Chiapolino, Richard Saurel. Models and methods for two-layer shallow water flows. Journal of Computational Physics, 2018, 371, pp.1043-1066. 10.1016/j.jcp.2018.05.034 hal-01809202

\section{HAL Id: hal-01809202 \\ https://hal-amu.archives-ouvertes.fr/hal-01809202}

Submitted on 6 Jun 2018

HAL is a multi-disciplinary open access archive for the deposit and dissemination of scientific research documents, whether they are published or not. The documents may come from teaching and research institutions in France or abroad, or from public or private research centers.
L'archive ouverte pluridisciplinaire HAL, est destinée au dépôt et à la diffusion de documents scientifiques de niveau recherche, publiés ou non, émanant des établissements d'enseignement et de recherche français ou étrangers, des laboratoires publics ou privés. 


\title{
Models and methods for two-layer shallow water flows
}

\author{
Alexandre Chiapolino ${ }^{1 \mathrm{a}, \mathrm{b}}$, Richard Saurel ${ }^{2 \mathrm{a}, \mathrm{b}, \mathrm{c}}$ \\ ${ }^{a}$ Aix Marseille Univ, CNRS, Centrale Marseille, LMA, Marseille, France \\ ${ }^{b}$ RS2N, Chemin de Gaumin, Saint-Zacharie 83640, France \\ ${ }^{c} I U F$, University Institute of France
}

\begin{abstract}
Two-layer shallow water models present at least two fundamental difficulties that are addressed in the present contribution. The first one is related to the lack of hyperbolicity of most existing models. By considering weak compressibility of the phases, a strictly hyperbolic formulation with pressure relaxation is obtained. It is shown to tend to the conventional two-layer model in the stiff pressure relaxation limit. The second issue is related to the non-conservative terms in the momentum equations. Analyzing the Riemann problem structure, local constants appear precisely at locations where the non-conservative products need definition. Thanks to these local constants, a locally conservative formulation of the equations is obtained, simplifying the Riemann problem resolution through a HLL-type Riemann solver. The method is compared to literature data, showing accurate and oscillation free solutions. Additional numerical experiments show robustness and accuracy of the method.

Keywords: multiphase flows, shallow water, hyperbolic systems, Riemann problem, non-conservative, relaxation.
\end{abstract}

\footnotetext{
${ }^{1}$ Corresponding author: alexandre.chiapolino@rs2n.eu

${ }^{2}$ richard.saurel@univ-amu.fr
} 


\section{Introduction}

Two-layer (and multi-layer) shallow water models are particularly useful in some limit cases of multi-fluid and variable density flows separated by nearly horizontal interfaces. These models govern the dynamics of incompressible fluids spreading under gravity effects. It can be for example:

- Flows of the same liquid but at different temperatures, resulting in density differences, such situation being typical of oceanic flows;

- Flows of two liquids of different densities;

- Flows of two gases evolving at low Mach number.

The two-layer approach is particularly interesting compared to multidimensional approaches, that consider vertical motion, as it enables much faster computations. It is also helpful when the height of one of the phases is arbitrarily small, as there is no need to spatially resolve it. Thereby, no numerical diffusion of the nearly horizontal interface is present and no interface tracking is needed. However, there are obviously some limitations with this approach:

- The vertical velocity component is neglected;

- The velocity is assumed uniform in cross sections of each layer.

Such type of modeling also involves serious difficulties. Indeed, most models are not hyperbolic, this issue having serious consequences both for propagation phenomenon, which becomes ill-posed, and for the design of numerical methods. A second serious difficulty appears as nonconservative terms are present in the momentum equations. The present paper addresses these two difficulties and provides solutions.

In the frame of averaged (or homogenized) equations in fluid mechanics, the issue related to the lack of hyperbolicity appears in different type of models, such as those of non-equilibrium two-phase flows. Only a few models seem well-posed with this respect, (Marble (1963) [1], Baer and Nunziato (1986) [2], Saurel et al. (2017) [3]). There are mainly two types of remedy to cure this issue: 
- Consider compressibility of the phases and deal with pressure relaxation [4]. This approach involves sound propagation in the phases and is particularly efficient in many situations. It has been adopted in the last two above-mentioned references.

- Consider turbulent effects in the phases, as they result in the appearance of a "turbulent sound speed" (Forestier et al. (1997) [5], Saurel et al. (2003) [6], Lhuillier et al. (2013) [7]). In the frame of shallow water flows, these effects have been studied in Richard and Gavrilyuk (2012) [8] and Gavrilyuk et al. (2016) [9].

In the present work, the first method is adopted and the fluids are considered weakly compressible. The resulting model is strictly hyperbolic and in the limit of stiff pressure relaxation, the conventional (non-hyperbolic) two-layer model is recovered. This approach is reminiscent of the model of Abgrall and Karni (2009) [10], except that extra pressure terms are present in the momentum equations of the new formulation. It also gives another interpretation of the relaxation approach, now based on compressibility and pressure effects.

The second issue is addressed as well and is related to the presence of non-conservative terms in the momentum equations. By examining the Riemann problem structure, it appears that local constants are present, at locations where the derivative of the Heaviside function emerges. Consequently the non-conservative products become well-defined. Also, local conservation laws are obtained and used in the frame of HLL-type Riemann solver.

The accuracy of the new solver is checked against results of Abgrall and Karni (2009) [10] as well as results obtained with a flow solver based on the VFRoe method of Gallouet and Masella (1996) [11] as it is able to deal, to some extent, with both conservative and non-conservative systems. The new method, based on HLL-type solver shows results of high accuracy and is oscillation free.

The paper is organized as follows. The two-layer hyperbolic model is presented in Section 2 and its stiff mechanical relaxation limit is examined. Both approximate VFRoe solver and non-conservative HLL solver are considered in Section 3. A Godunov-type scheme is derived in the same section. Results and validations are addressed in Sections 4 and 5 . Conclusions are given in Section 6. 


\section{Hyperbolic two-layer shallow water model}

The conventional two-layer shallow water model (Ovsyannikov (1979) [12]) reads,

$$
\left\{\begin{array}{l}
\frac{\partial\left(h_{1} \rho_{1}\right)}{\partial t}+\frac{\partial\left(h_{1} \rho_{1} u_{1}\right)}{\partial x}=0 \\
\frac{\partial\left(h_{1} \rho_{1} u_{1}\right)}{\partial t}+\frac{\partial\left(h_{1} \rho_{1} u_{1}^{2}+\frac{1}{2} \rho_{1} g h_{1}^{2}+g \rho_{2} h_{1} h_{2}\right)}{\partial x}=\rho_{2} g h_{2} \frac{\partial h_{1}}{\partial x} \\
\frac{\partial\left(h_{2} \rho_{2}\right)}{\partial t}+\frac{\partial\left(h_{2} \rho_{2} u_{2}\right)}{\partial x}=0 \\
\frac{\partial\left(h_{2} \rho_{2} u_{2}\right)}{\partial t}+\frac{\partial\left(h_{2} \rho_{2} u_{2}^{2}+\frac{1}{2} \rho_{2} g h_{2}^{2}\right)}{\partial x}=-\rho_{2} g h_{2} \frac{\partial h_{1}}{\partial x}
\end{array}\right.
$$

$h_{1}$ and $h_{2}$ denote the heights of the two layers, $\rho_{1}$ and $\rho_{2}$ represent the densities of the fluids, considered constant at this level, $u_{1}$ and $u_{2}$ denote the fluid velocities, averaged in each layer and $g$ represents the gravity constant. Topography effects have been omitted for the sake of simplicity as well as friction with the bottom and between layers.

System (2.1) has been examined in Abgrall and Karni (2009) [10], Kurganov and Petrova (2009) [13] and Monjarret (2015) [14] and appeared hyperbolic for small velocity drift only,

$$
\left(u_{1}-u_{2}\right)^{2}<\left(h_{1}+h_{2}\right) g\left(1-\frac{\rho_{2}}{\rho_{1}}\right) .
$$

Moreover the wave speeds can hardly be computed, rendering the system intricate to solve numerically. A method is given in Kurganov and Petrova (2009) [13] to overcome this difficulty. In the present approach, pressure non-equilibrium effects result in an unconditionally hyperbolic 
formulation:

$$
\left\{\begin{array}{l}
\frac{\partial h_{1}}{\partial t}+u_{1} \frac{\partial h_{1}}{\partial x}=\frac{\mu\left(p_{1}-p_{0}\right)}{\rho_{1} c_{1}^{2}}, \\
\frac{\partial\left(h_{1} \rho_{1}\right)}{\partial t}+\frac{\partial\left(h_{1} \rho_{1} u_{1}\right)}{\partial x}=0, \\
\frac{\partial\left(h_{1} \rho_{1} u_{1}\right)}{\partial t}+\frac{\partial\left(h_{1} \rho_{1} u_{1}^{2}+h_{1} p_{1}\left(\rho_{1}\right)+\frac{1}{2} \rho_{1} g h_{1}^{2}+g \rho_{2} h_{1} h_{2}\right)}{\partial x}=\rho_{2} g h_{2} \frac{\partial h_{1}}{\partial x}+p_{0} \frac{\partial h_{1}}{\partial x} \\
\frac{\partial h_{2}}{\partial t}+u_{2} \frac{\partial h_{2}}{\partial x}=\frac{\mu\left(p_{2}-p_{0}\right)}{\rho_{2} c_{2}^{2}}, \\
\frac{\partial\left(h_{2} \rho_{2}\right)}{\partial t}+\frac{\partial\left(h_{2} \rho_{2} u_{2}\right)}{\partial x}=0, \\
\frac{\partial\left(h_{2} \rho_{2} u_{2}\right)}{\partial t}+\frac{\partial\left(h_{2} \rho_{2} u_{2}^{2}+h_{2} p_{2}\left(\rho_{2}\right)+\frac{1}{2} \rho_{2} g h_{2}^{2}\right)}{\partial x}=-\rho_{2} g h_{2} \frac{\partial h_{1}}{\partial x}+p_{0} \frac{\partial h_{2}}{\partial x} .
\end{array}\right.
$$

Two equations have been added and express the transport of the heights of the fluid layers that are assumed to vary as a function of pressure differentials $\left(p_{k}-p_{0}\right) . p_{k}$ denotes the thermodynamic pressure of fluid $k$, given by barotropic (and convex) equations of state $p_{k}\left(\rho_{k}\right)$. Example of such equation of state (EOS) is,

$$
p_{k}\left(\rho_{k}\right)=p_{k}^{(0)}+c_{k}^{2}\left(\rho_{k}-\rho_{k}^{(0)}\right)
$$

with $k=1,2$. Other options, such as Tait EOS for instance are possible. We will see that the choice of the EOS is not important, only the related sound speed $c_{k}$ has importance. $p_{0}$ denotes the (constant) atmospheric pressure and $p_{k}^{(0)}=p_{0}$.

The assumption of constant atmospheric pressure is quite realistic when dealing with flows of gases having different densities. When dealing with liquids and large hydrostatic effects, the model can be reformulated with extra pressure terms as a function of heights of the fluid layers, and does not pose extra fundamental issues than those already addressed in the present contribution. Such extension is given in Appendix, but for the sake of simplicity of the presentation, this extended model is not considered in the rest of the paper.

The pressure relaxation parameter $\mu$ is related to the fluid sound speeds and heights of layers. It controls the rate at which pressure equilibrium is reached. Following Saurel et al. (2017) [3], the first equation of System (2.3) can be written as, 


$$
\frac{d_{1} h_{1}}{d t}=\frac{h_{1}}{\tau} \frac{p_{1}-p_{0}}{\rho_{1} c_{1}^{2}}
$$

where $\frac{d_{1}}{d t}=\frac{\partial}{\partial t}+u_{1} \frac{\partial}{\partial x}$ and $\tau$ is the pressure relaxation time,

$$
\tau=\frac{h_{1}}{c_{1}}
$$

corresponding to the following pressure relaxation parameter estimate:

$$
\mu \simeq \frac{h_{1}}{\tau} \simeq c_{1}
$$

In most situations, this relaxation time is of the order of $\frac{1}{100}$ second, meaning that the relaxation parameter $\mu$ is large: $\mu \simeq \operatorname{Max}\left(\tau_{1}^{-1}, \tau_{2}^{-1}\right)$ or alternatively $\mu \simeq \operatorname{Min}\left(c_{1}, c_{2}\right)$. In practical computations, the relaxation time $\tau$ will be assumed of the same order as the computational time step and stiff pressure relaxation will be done at the end of each time step. Therefore, there is no need of precise knowledge of the pressure relaxation parameter $\mu$.

This system is reminiscent of Baer and Nunziato's (1986) [2] model widely used in two-phase flow modeling. It is also reminiscent of Abgrall and Karni's (2009) [10] relaxation model, except that pressure terms have been added to the momentum equations $\left(h_{1} p_{1}\left(\rho_{1}\right)\right.$ and $\left.h_{2} p_{2}\left(\rho_{2}\right)\right)$. To maintain mechanical equilibrium, extra non-conservative terms have been added in the righthand side $\left(p_{0} \frac{\partial h_{k}}{\partial x}\right)$. These terms are not in contradiction with the total momentum conservation that reads:

$$
\begin{aligned}
& \frac{\partial\left(h_{1} \rho_{1} u_{1}+h_{2} \rho_{2} u_{2}\right)}{\partial t} \\
& +\frac{\partial\left[h_{1} \rho_{1} u_{1}^{2}+h_{1} p_{1}\left(\rho_{1}\right)+\frac{1}{2} \rho_{1} g h_{1}^{2}+g \rho_{2} h_{1} h_{2}+h_{2} \rho_{2} u_{2}^{2}+h_{2} p_{2}\left(\rho_{2}\right)+\frac{1}{2} \rho_{2} g h_{2}^{2}-p_{0}\left(h_{1}+h_{2}\right)\right]}{\partial x}=0
\end{aligned}
$$

Let us now examine some relevant properties to check validity of this formulation. 


\subsection{Hyperbolicity}

System (2.3) is expressed in primitive-variable formulation (in the absence of source terms) as,

$$
\frac{\partial W}{\partial t}+A(W) \frac{\partial W}{\partial x}=0
$$

with

$$
W=\left(\begin{array}{c}
h_{1} \\
\rho_{1} \\
u_{1} \\
h_{2} \\
\rho_{2} \\
u_{2}
\end{array}\right), \quad A(W)=\left(\begin{array}{cccccc}
u_{1} & 0 & 0 & 0 & 0 & 0 \\
0 & u_{1} & \rho_{1} & 0 & 0 & 0 \\
\frac{p_{1}-p_{0}}{h_{1} \rho_{1}}+g & \frac{c_{1}^{2}+\frac{1}{2} g h_{1}}{\rho_{1}} & u_{1} & g \frac{\rho_{2}}{\rho_{1}} & \frac{g h_{2}}{\rho_{1}} & 0 \\
0 & 0 & 0 & u_{2} & 0 & 0 \\
0 & 0 & 0 & 0 & u_{2} & \rho_{2} \\
g & 0 & 0 & \frac{p_{2}-p_{0}}{h_{2} \rho_{2}}+g & \frac{c_{2}^{2}+\frac{1}{2} g h_{2}}{\rho_{2}} & u_{2}
\end{array}\right) .
$$

The wave speeds are solutions of $\operatorname{det}(A(W)-\lambda \underline{\underline{I}})=0$ resulting in,

$$
\left(u_{1}-\lambda\right)\left(u_{2}-\lambda\right)\left[\left(u_{2}-\lambda\right)^{2}-\left(c_{2}^{2}+\frac{1}{2} g h_{2}\right)\right]\left[\left(u_{1}-\lambda\right)^{2}-\left(c_{1}^{2}+\frac{1}{2} g h_{1}\right)\right]=0 .
$$

Six real and distinct eigenvalues appear as:

$$
\left\{\begin{array}{lll}
\lambda_{1}=u_{1}, & \lambda_{2}=u_{1}+\sqrt{c_{1}^{2}+\frac{1}{2} g h_{1}}, & \lambda_{3}=u_{1}-\sqrt{c_{1}^{2}+\frac{1}{2} g h_{1}}, \\
\lambda_{4}=u_{2}, & \lambda_{5}=u_{2}+\sqrt{c_{2}^{2}+\frac{1}{2} g h_{2}}, & \lambda_{6}=u_{2}-\sqrt{c_{2}^{2}+\frac{1}{2} g h_{2} .}
\end{array}\right.
$$

Those eigenvalues correspond to the wave speeds emerging at a given initial discontinuity, as schematized in Fig. 1,

System (2.3) is consequently strictly hyperbolic. This model is however relevant with respect to the physics expressed in (2.1) if it tends to the same equations when pressure relaxation is stiff. This limit is examined hereafter. 


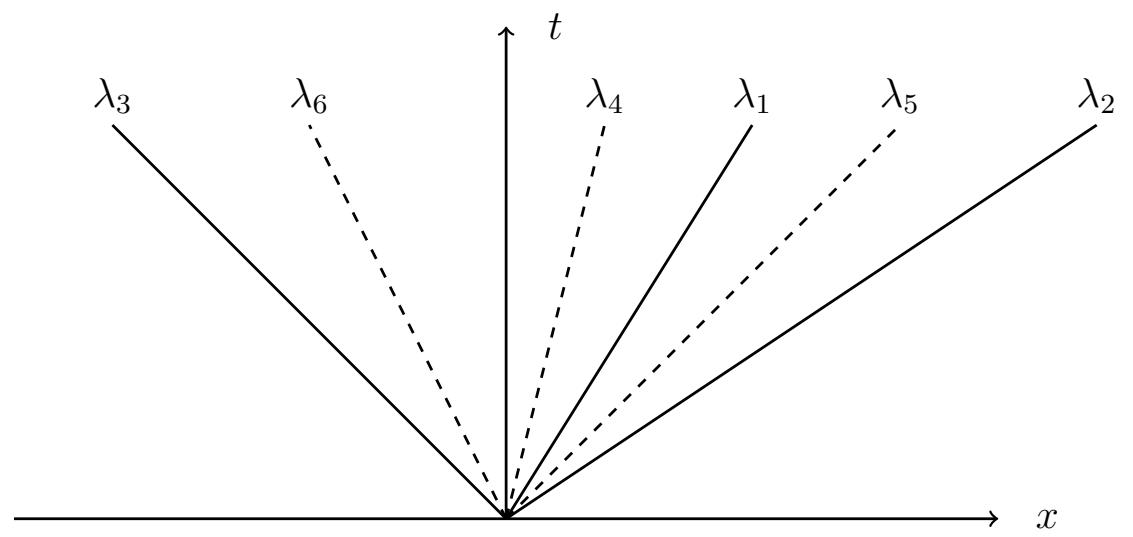

Figure 1: Schematic representation in the $(\mathrm{x}, \mathrm{t})$ diagram of the six waves (2.10) present in the flow model (2.3) and emerging at a given initial discontinuity.

\subsection{Stiff pressure relaxation limit}

The mass and height equations of a given phase are analyzed:

$$
\left\{\begin{array}{l}
\frac{\partial h_{k}}{\partial t}+u_{k} \frac{\partial h_{k}}{\partial x}=\frac{\mu\left(p_{k}-p_{0}\right)}{\rho_{k} c_{k}^{2}}, \\
\frac{\partial\left(h_{k} \rho_{k}\right)}{\partial t}+\frac{\partial\left(h_{k} \rho_{k} u_{k}\right)}{\partial x}=0,
\end{array}\right.
$$

with $k=1,2$. Their combination results in,

$$
\frac{d_{k} \rho_{k}}{d t}+\rho_{k} \frac{\partial u_{k}}{\partial x}=-\frac{\rho_{k}}{h_{k}} \frac{\mu\left(p_{k}-p_{0}\right)}{\rho_{k} c_{k}^{2}} .
$$

Inserting the phase $k$ equation of state $p_{k}\left(\rho_{k}\right)$, the following pressure evolution equation is obtained,

$$
\frac{d_{k} p_{k}}{d t}+\rho_{k} c_{k}^{2} \frac{\partial u_{k}}{\partial x}=-\frac{\mu\left(p_{k}-p_{0}\right)}{h_{k}} .
$$

As the atmospheric pressure $p_{0}$ is constant, the last equation can be expressed as,

$$
\frac{d_{k}\left(p_{k}-p_{0}\right)}{d t}+\rho_{k} c_{k}^{2} \frac{\partial u_{k}}{\partial x}=-\frac{\mu\left(p_{k}-p_{0}\right)}{h_{k}} .
$$

The phase pressure is expressed around the equilibrium state with the following expansion,

$$
p_{k}=\left(p_{k}^{(0)}+\epsilon p_{k}^{(1)}+\ldots\right),
$$


where

$-\epsilon$ is of the order of the inverse of pressure relaxation parameter $\left(\epsilon \simeq \mu^{-1} \simeq \tau\right)$, tending to zero in most situations $\left(\epsilon \rightarrow 0^{+}\right)$as discussed earlier (see also Kapila et al. (2001) [15] for estimates in the context of granular flows),

$-p_{k}^{(0)}$ and $p_{k}^{(1)}$ represent respectively the leading and first-order pressure terms of the Taylor expansion.

Inserting these definitions in the pressure evolution equation,

$$
\frac{d_{k}\left(\left[p_{k}^{(0)}+\epsilon p_{k}^{(1)}+\ldots\right]-p_{0}\right)}{d t}+\rho_{k} c_{k}^{2} \frac{\partial u_{k}}{\partial x}=-\frac{\left[p_{k}^{(0)}+\epsilon p_{k}^{(1)}+\ldots\right]-p_{0}}{h_{k} \epsilon},
$$

the following results are obtained:

- At leading order $\left(\epsilon^{-1}\right): p_{k}^{(0)}=p_{0}$;

- At first order $p_{k}^{(1)}=-\rho_{k} c_{k}^{2} h_{k} \frac{\partial u_{k}}{\partial x}$.

Inserting this last result in the height equations,

$$
\frac{\partial h_{k}}{\partial t}+u_{k} \frac{\partial h_{k}}{\partial x}=\frac{\mu\left(p_{k}-p_{0}\right)}{\rho_{k} c_{k}^{2}} \simeq \frac{p_{k}^{(1)}}{\rho_{k} c_{k}^{2}} \simeq-h_{k} \frac{\partial u_{k}}{\partial x}
$$

they become,

$$
\frac{\partial h_{k}}{\partial t}+\frac{\partial\left(h_{k} u_{k}\right)}{\partial x} \simeq 0
$$

The mass equations are unchanged while modifications in the momentum equations appear as a consequence of the equilibrium condition $\left(p_{k}=p_{0}\right)$. They finally result at leading order in,

$$
\left\{\begin{array}{l}
\frac{\partial\left(h_{1} \rho_{1} u_{1}\right)}{\partial t}+\frac{\partial\left(h_{1} \rho_{1} u_{1}^{2}+\frac{1}{2} \rho_{1} g h_{1}^{2}+g \rho_{2} h_{1} h_{2}\right)}{\partial x}=\rho_{2} g h_{2} \frac{\partial h_{1}}{\partial x} \\
\frac{\partial\left(h_{2} \rho_{2} u_{2}\right)}{\partial t}+\frac{\partial\left(h_{2} \rho_{2} u_{2}^{2}+\frac{1}{2} \rho_{2} g h_{2}^{2}\right)}{\partial x}=-\rho_{2} g h_{2} \frac{\partial h_{1}}{\partial x} .
\end{array}\right.
$$

System (2.1) is recovered, complemented by two conservation equations for the heights, that are in agreement with the two mass equations as soon as the densities are constants. 
It thus appears that System (2.3) tends to System (2.1) when pressure relaxation is stiff. As System (2.3) is hyperbolic, it is a good candidate to approximate (2.1) numerically with a two-step procedure:

- Solve the hyperbolic system (2.3) without source terms;

- Relax the pressures onto the atmospheric one and reset the heights.

This is similar to the method of Saurel and Abgrall (1999) [16] to compute flows with interfaces separating fluids. Before entering in the details of the hyperbolic solver, let us present the pressure relaxation process, that is particularly simple in the present context.

\subsection{Stiff pressure relaxation solver}

Let us consider for example EOS (2.4). Consequently, the densities as functions of pressures are given by:

$$
\rho_{k}=\rho_{k}^{(0)}+\frac{p_{k}-p_{k}^{(0)}}{c_{k}^{2}} .
$$

As the pressures relax to the atmospheric one $\left(p_{k}=p_{k}^{(0)}=p_{0}\right)$, the densities at relaxed pressure are just,

$$
\rho_{k}^{*}=\rho_{k}^{(0)},
$$

where the superscript ${ }^{*}$ denotes the relaxed pressure state. As the masses of each layer are computed by associated mass balance equations and are constant during the relaxation process,

$$
m_{k}=h_{k} \rho_{k}=h_{k}^{*} \rho_{k}^{*}
$$

the heights at relaxed states are reset as,

$$
h_{k}^{*}=\frac{h_{k} \rho_{k}}{\rho_{k}^{(0)}} .
$$

The stiff pressure relaxation solver just consists in the reset of the heights of the fluids $h_{k} \rightarrow h_{k}^{*}$ and is independent of the equations of state. At this level, the relaxation method of Abgrall and Karni (2009) [10] is recovered. We now address the design of hyperbolic solvers. 


\section{Approximate Riemann solvers}

Two different approximate methods are considered to solve the Riemann problem of System (2.3), the VFRoe method (Gallouet and Masella (1996) [11]) and a new HLL-type Riemann solver (Harten et al. (1983) [17]). As System (2.3) contains non-conservative terms, an approach dealing with both conservative and non-conservative systems is examined first.

\subsection{VFRoe solver}

The VFRoe method considers the equations in non-conservative formulation,

$$
\frac{\partial W}{\partial t}+A(\bar{W}) \frac{\partial W}{\partial x}=0
$$

with

$$
W=\left(h_{1}, \rho_{1}, u_{1}, h_{2}, \rho_{2}, u_{2}\right)^{T} \quad \text { and } \quad \bar{W}=\frac{W_{L}+W_{R}}{2},
$$

where $W_{L}$ and $W_{R}$ are respectively the left and right-state vectors at a given cell boundary.

The VFRoe method considers the exact Riemann problem solution of (3.1). Note that (3.1) is a local linearization of the non-linear flow model (2.3) around state $\bar{W}$. The VFRoe solution is thus the exact solution of an approximate problem.

The exact solution of (3.1) may be found in many textbooks related to hyperbolic systems (LeVeque (2002) [18], Toro (2013) [19]) and can be summarized as follows,

$$
W^{*}=W_{L}+\sum_{\overline{\lambda_{i}}<0} \overline{a_{i}} \overline{R_{i}}=W_{R}-\sum_{\overline{\lambda_{i}}>0} \overline{a_{i}} \overline{R_{i}},
$$

where the wave strengths $\overline{a_{i}}$ are the coefficients resulting from the decomposition of the eigenvectors.

$$
W_{R}-W_{L}=\sum_{\overline{\lambda_{i}}} \overline{a_{i}} \overline{R_{i}}
$$

For the sake of space restriction, the right eigenvectors $R_{i}$ and the wave strengths $\overline{a_{i}}$ are not detailed, associated formulas being considerably large. The main weakness of this method is 
related to the average $\bar{W}$ which can be far from the solution of the non-linear problem, resulting in positivity issues especially when large amplitude waves are present.

With the help of the Riemann problem solution (3.2), the various equations of System (2.3) are updated with a Godunov-type method (stable under the conventional $C F L$ condition) as,

$$
\begin{gathered}
h_{k}^{n+1}=h_{k}^{n}-\frac{\Delta t}{\Delta x}\left(\left(h_{k} u_{k}\right)_{i+\frac{1}{2}}^{*}-\left(h_{k} u_{k}\right)_{i-\frac{1}{2}}^{*}\right)+\frac{\Delta t}{\Delta x} h_{k, i}^{n}\left(u_{k, i+\frac{1}{2}}^{*}-u_{k, i-\frac{1}{2}}^{*}\right), \\
\left(h_{k} \rho_{k}\right)_{i}^{n+1}=\left(h_{k} \rho_{k}\right)_{i}^{n}-\frac{\Delta t}{\Delta x}\left(\left(h_{k} \rho_{k} u_{k}\right)_{i+\frac{1}{2}}^{*}-\left(h_{k} \rho_{k} u_{k}\right)_{i-\frac{1}{2}}^{*}\right), \\
\left(h_{1} \rho_{1} u_{1}\right)_{i}^{n+1}=\left(h_{1} \rho_{1} u_{1}\right)_{i}^{n}-\frac{\Delta t}{\Delta x}\left\{\left(h_{1} \rho_{1} u_{1}^{2}+h_{1}\left(p_{1}-p_{0}\right)+\frac{1}{2} \rho_{1} h_{1}^{2}\right)_{i+\frac{1}{2}}^{*}\right. \\
\left.-\left(h_{1} \rho_{1} u_{1}^{2}+h_{1}\left(p_{1}-p_{0}\right)+\frac{1}{2} \rho_{1} h_{1}^{2}\right)_{i-\frac{1}{2}}^{*}\right\}+\frac{\Delta t}{\Delta x} h_{1, i}^{n}\left\{\left(-\rho_{2} g h_{2}\right)_{i+\frac{1}{2}}^{*}-\left(-\rho_{2} g h_{2}\right)_{i-\frac{1}{2}}^{*}\right\} \\
\left(h_{2} \rho_{2} u_{2}\right)_{i}^{n+1}=\left(h_{2} \rho_{2} u_{2}\right)_{i}^{n}-\frac{\Delta t}{\Delta x}\left\{\left(h_{2} \rho_{2} u_{2}^{2}+h_{2}\left(p_{2}-p_{0}\right)+\frac{1}{2} \rho_{2} h_{2}^{2}+\rho_{2} g h_{1} h_{2}\right)_{i+\frac{1}{2}}^{*}\right. \\
\left.-\left(h_{2} \rho_{2} u_{2}^{2}+h_{2}\left(p_{2}-p_{0}\right)+\frac{1}{2} \rho_{2} h_{2}^{2}+\rho_{2} g h_{1} h_{2}\right)_{i-\frac{1}{2}}^{*}\right\} \\
+\frac{\Delta t}{\Delta x} h_{1, i}^{n}\left\{\left(\left(\rho_{2} g h_{2}\right)_{i+\frac{1}{2}}^{*}-\left(\rho_{2} g h_{2}\right)_{i-\frac{1}{2}}^{*}\right)\right\},
\end{gathered}
$$

where $n+1$ and $n$ denote two consecutive time steps and superscript ${ }^{*}$ denotes the VFRoe Riemann problem solution given by Eq. (3.2). Indexes $i$ and $i \pm \frac{1}{2}$ denote respectively the center of the current numerical cell and its corresponding boundaries.

\subsection{HLL-type Riemann solver}

Let us consider a simplified solver, based on Rankine-Hugoniot conditions, such as the HLL solver. In this frame, the two extreme waves $S_{L}$ and $S_{R}$ are approximated following Davis (1988) 
[20] as,

$$
\left\{\begin{array}{l}
S_{L, k}=\min \left(u_{L, k}-\sqrt{c_{L, k}^{2}+\frac{1}{2} g h_{L, k}}, u_{R, k}-\sqrt{c_{R, k}^{2}+\frac{1}{2} g h_{R, k}}\right) \\
S_{R, k}=\max \left(u_{L, k}+\sqrt{c_{L, k}^{2}+\frac{1}{2} g h_{L, k}}, u_{R, k}+\sqrt{c_{R, k}^{2}+\frac{1}{2} g h_{R, k}}\right)
\end{array}\right.
$$

with $k=1,2$. The indexes $L_{L}$ and ${ }_{R}$ denote respectively the left and right states at a given cell boundary. The two extreme waves are considered as,

$$
S_{L}=\min \left(S_{L, 1}, S_{L, 2}\right), \quad S_{R}=\max \left(S_{R, 1}, S_{R, 2}\right)
$$

The two contact waves $u_{1}$ and $u_{2}$ are considered as well for the transport of the heights $h_{1}$ and $h_{2}$, as depicted in Fig. 2 ,

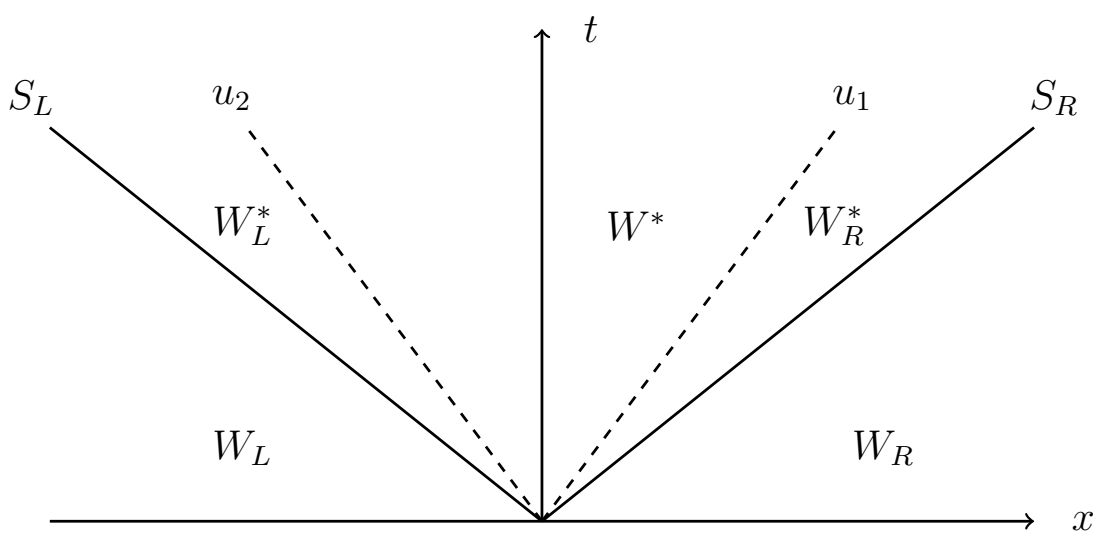

Figure 2: Schematic representation in the $(x, t)$ diagram of the two extreme waves and the two contact waves considered for the transport of the two heights.

Regarding the transport equations, the exact Riemann problem solution is straightforward:

$$
\begin{cases}h_{1}^{*}\left(\frac{x}{t}<u_{1}^{*}\right)=h_{1, L}, & h_{1}^{*}\left(\frac{x}{t}>u_{1}^{*}\right)=h_{1, R}, \\ h_{2}^{*}\left(\frac{x}{t}<u_{2}^{*}\right)=h_{2, L}, \quad h_{2}^{*}\left(\frac{x}{t}>u_{2}^{*}\right)=h_{2, R} .\end{cases}
$$

These solutions indicate that the non-conservative terms have contributions between the two extreme waves $S_{R}$ and $S_{L}$, at points where $h_{1}$ and $h_{2}$ are discontinuous. More precisely, only the discontinuity in $h_{1}$ needs attention, as the non-conservative terms involving the atmospheric pressure (considered constant) transform to fluxes, 


$$
p_{0} \frac{\partial h_{k}}{\partial x}=\frac{\partial\left(p_{0} h_{k}\right)}{\partial x}
$$

It thus remains to analyze only the non-conservative term,

$$
\rho_{2} g h_{2} \frac{\partial h_{1}}{\partial x}
$$

The solution states for $\left(\rho_{2} h_{2}\right)$ are given by,

$$
\left(\rho_{2} h_{2}\right)_{L}^{*}=\left(\rho_{2} h_{2}\right)_{L} \frac{u_{2, L}-S_{L}}{u_{2}^{*}-S_{L}} \quad \text { and } \quad\left(\rho_{2} h_{2}\right)_{R}^{*}=\left(\rho_{2} h_{2}\right)_{R} \frac{u_{2, R}-S_{R}}{u_{2}^{*}-S_{R}}
$$

A schematic representation is given in Fig. 3.

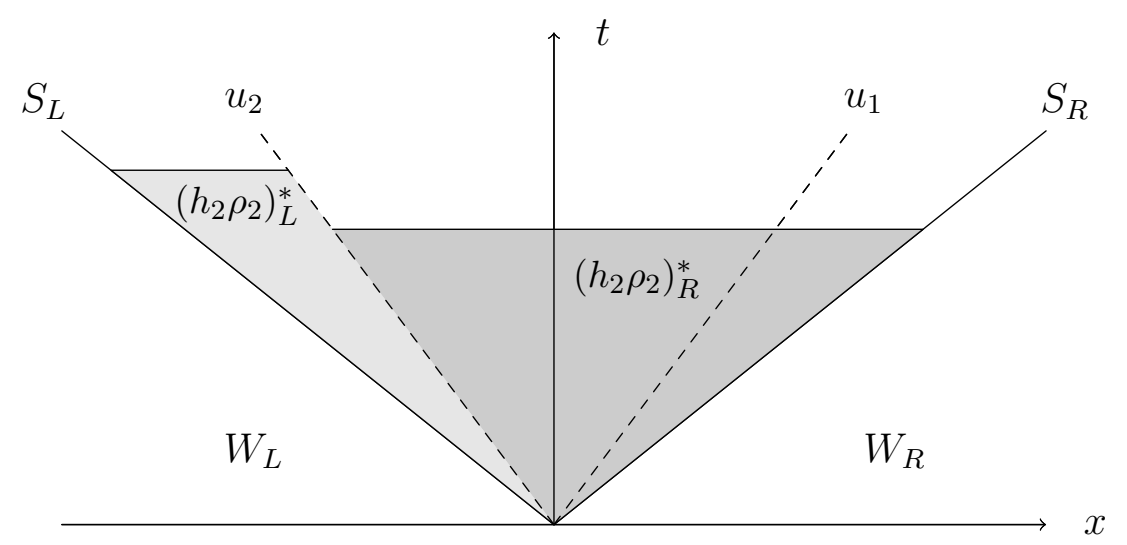

Figure 3: Schematic representation in the $(\mathrm{x}, \mathrm{t})$ diagram of the two levels $\left(\rho_{2} h_{2}\right)_{L, R}^{*}$ in the Riemann problem solution.

These formulas need $u_{2}^{*}$ for their practical use that is unknown at this level. However, according to the sign of the velocity difference $u_{1}^{*}-u_{2}^{*}$, only two instances may occur:

- If $u_{1}^{*}>u_{2}^{*}$, the $\rho_{2} g h_{2} \frac{\partial h_{1}}{\partial x}$ term becomes locally $g\left(\rho_{2} h_{2}\right)_{R}^{*} \frac{\partial h_{1}}{\partial x}$. As $\left(\rho_{2} h_{2}\right)_{R}^{*}$ is constant at the point where $\frac{\partial h_{1}}{\partial x}$ is discontinuous, the non-conservative term becomes locally;

$$
\rho_{2} g h_{2} \frac{\partial h_{1}}{\partial x}=\frac{\partial\left[g\left(\rho_{2} h_{2}\right)_{R}^{*} h_{1}\right]}{\partial x} .
$$

- If $u_{1}^{*}<u_{2}^{*}$, the same reasoning yields,

$$
\rho_{2} g h_{2} \frac{\partial h_{1}}{\partial x}=\frac{\partial\left[g\left(\rho_{2} h_{2}\right)_{L}^{*} h_{1}\right]}{\partial x} .
$$


It thus appears that the momentum equations are locally conservative. However, their explicit determination and use require knowledge of both $u_{1}^{*}$ and $u_{2}^{*}$ that are themselves solutions of integration of the momentum equations.

To simplify the algorithm, a single solution state is considered for the apparent densities $\left(\rho_{k} h_{k}\right)^{*}$ instead of the two $\left(\rho_{k} h_{k}\right)_{L}^{*}$ and $\left(\rho_{k} h_{k}\right)_{R}^{*}$ in the same mind as in the HLL solver for the Euler equations:

$$
\left(h_{k} \rho_{k}\right)^{*}=\frac{\left(h_{k} \rho_{k}\right)_{R}\left(u_{k, R}-S_{R}\right)-\left(h_{k} \rho_{k}\right)_{L}\left(u_{k, L}-S_{L}\right)}{S_{L}-S_{R}} .
$$

Thanks to this approximation, the momentum equations become locally,

$$
\left\{\begin{array}{l}
\frac{\partial\left(h_{1} \rho_{1} u_{1}\right)}{\partial t}+\frac{\partial\left(h_{1} \rho_{1} u_{1}^{2}+h_{1}\left[p_{1}\left(\rho_{1}\right)-p_{0}\right]+\frac{1}{2} \rho_{1} g h_{1}^{2}+g \rho_{2} h_{1} h_{2}-g\left(h_{2} \rho_{2}\right)^{*} h_{1}\right)}{\partial x}=0 \\
\frac{\partial\left(h_{2} \rho_{2} u_{2}\right)}{\partial t}+\frac{\partial\left(h_{2} \rho_{2} u_{2}^{2}+h_{2}\left[p_{2}\left(\rho_{2}\right)-p_{0}\right]+\frac{1}{2} \rho_{2} g h_{2}^{2}+g\left(h_{2} \rho_{2}\right)^{*} h_{1}\right)}{\partial x}=0
\end{array}\right.
$$

Denoting the momentum fluxes by,

$$
\left\{\begin{array}{l}
F_{1, \text { mom }}=h_{1} \rho_{1} u_{1}^{2}+h_{1}\left[p_{1}\left(\rho_{1}\right)-p_{0}\right]+\frac{1}{2} \rho_{1} g h_{1}^{2}+g \rho_{2} h_{1} h_{2}-g\left(h_{2} \rho_{2}\right)^{*} h_{1}, \\
F_{2, \text { mom }}=h_{2} \rho_{2} u_{2}^{2}+h_{2}\left[p_{2}\left(\rho_{2}\right)-p_{0}\right]+\frac{1}{2} \rho_{2} g h_{2}^{2}+g\left(h_{2} \rho_{2}\right)^{*} h_{1},
\end{array}\right.
$$

the momentum numerical fluxes are then given by,

$$
F_{k, \text { mom }}^{*}=\frac{F_{k, m o m, R} S_{L}-F_{k, m o m, L} S_{R}+S_{L} S_{R}\left(U_{k, m o m, L}-U_{k, m o m, R}\right)}{S_{L}-S_{R}},
$$

with $U_{k, m o m}=h_{k} \rho_{k} u_{k}$. The mass numerical fluxes are computed by the HLL approximation as well and read,

$$
F_{k, \text { mass }}^{*}=\frac{\left(h_{k} \rho_{k}\right)_{R} S_{L}\left(u_{k, R}-S_{R}\right)-\left(h_{k} \rho_{k}\right)_{L} S_{R}\left(u_{k, L}-S_{L}\right)}{S_{L}-S_{R}} .
$$

System (2.1) being non-conservative, the conservative variable-state vector and in particular the fluid velocities are also needed for the computations. With the help of Eqs. (3.12) and (3.13), 
the momentum variables are computed with the HLL approximation as,

$$
\left(h_{k} \rho_{k} u_{k}\right)^{*}=\frac{F_{k, \text { mom }, R}-F_{k, \text { mom }, L}-S_{R} U_{k, \text { mom }, R}+S_{L} U_{k, \text { mom }, L}}{S_{L}-S_{R}} .
$$

Using Eqs. (3.11) and (3.16), the speeds of the fluids are given by,

$$
u_{k}^{*}=\frac{\left(h_{k} \rho_{k} u_{k}\right)^{*}}{\left(h_{k} \rho_{k}\right)^{*}} .
$$

The associated Godunov-type method now reads,

$$
\left\{\begin{aligned}
h_{k}^{n+1} & =h_{k}^{n}-\frac{\Delta t}{\Delta x}\left((h u)_{k, i+\frac{1}{2}}^{*}-(h u)_{k, i-\frac{1}{2}}^{*}\right)+\frac{\Delta t}{\Delta x} h_{k, i}^{n}\left(u_{k, i+\frac{1}{2}}^{*}-u_{k, i-\frac{1}{2}}^{*}\right), \\
\left(h_{k} \rho_{k}\right)_{i}^{n+1} & =\left(h_{k} \rho_{k}\right)_{i}^{n}-\frac{\Delta t}{\Delta x}\left(F_{k, \text { mass }, i+\frac{1}{2}}^{*}-F_{k, \text { mass }, i-\frac{1}{2}}^{*}\right), \\
\left(h_{1} \rho_{1} u_{1}\right)_{i}^{n+1} & =\left(h_{1} \rho_{1} u_{1}\right)_{i}^{n}-\frac{\Delta t}{\Delta x}\left(F_{1, \text { mom }, i+\frac{1}{2}}^{*}-F_{1, \text { mom }, i-\frac{1}{2}}^{*}\right)+\frac{\Delta t}{\Delta x} h_{1, i}^{n}\left(-g\left[\left(h_{2} \rho_{2}\right)_{i+\frac{1}{2}}^{*}-\left(h_{2} \rho_{2}\right)_{i-\frac{1}{2}}^{*}\right]\right), \\
\left(h_{2} \rho_{2} u_{2}\right)_{i}^{n+1} & =\left(h_{2} \rho_{2} u_{2}\right)_{i}^{n}-\frac{\Delta t}{\Delta x}\left(F_{2, \text { mom }, i+\frac{1}{2}}^{*}-F_{2, \text { mom }, i-\frac{1}{2}}^{*}\right)+\frac{\Delta t}{\Delta x} h_{1, i}^{n}\left(g\left[\left(h_{2} \rho_{2}\right)_{i+\frac{1}{2}}^{*}-\left(h_{2} \rho_{2}\right)_{i-\frac{1}{2}}^{*}\right]\right) .
\end{aligned}\right.
$$

Efficiency of both VFRoe and HLL solvers are now investigated on various test problems of the literature.

\section{Results and validations}

It is important to address in priority the effects of the fluid EOS with the present relaxation approach. Indeed the model is hyperbolic as a consequence of compressibility terms in the momentum equations. Extra tests, where VFRoe and HLL solvers are compared, are addressed subsequently.

\subsection{Effects of the artificial sound speed}

The EOS (2.4) involves sound speed $c_{k}$ that has influence on computed results, as shown hereafter in Figs. 4, 5 and 6. The examined configuration consists in a limit case where the initial height of the first fluid (lower layer) is as low as numerically admissible, so that only the upper layer (second fluid) evolves significantly. With this specific configuration, the solution of 
the two-layer shallow water system (2.3) is meant to be compared to the exact solution of the one-layer Saint-Venant equations.

A dam-break problem is used to this end. The following test is proposed in LeVeque's textbook (2002) [18] to illustrate behavior of the solution of the Saint-Venant equations. It consists in a dam, separating two levels of fluids, that bursts at time $t=0$. All variables of the current test problem are in dimensionless units as done in [18]. This test is the shallow water equivalent of the shock-tube problem of gas dynamics and appears to be an excellent benchmark as the flow deals with shock and expansion waves that create arduous conditions. The constant gravity is normalized and reads $g=1$. The numerical domain has a length set to 10 with a height discontinuity initially located in the middle. On the left of this discontinuity, the fluid is initially at $h=3$ and $h=1$ on the right. The fluid is initially at rest on either side of the discontinuity.

To mimic the Saint-Venant system with the two-layer model (2.3), the height of the first fluid is initially set to $h_{1}=\epsilon=10^{-6}$ throughout the whole numerical domain. Its density is set to $\rho_{1}=1.2$ and its velocity is set to $u_{1}=0$. The second fluid, placed above the first one, has initial heights $h_{2}=3$ at left and $h_{2}=1$ at right. Its density is set to $\rho_{2}=1$ and its velocity is set to $u_{2}=0$.

Figure 4 shows the results with constant sound speed set to $c_{k}=100$. The same sound speed has been taken for both fluids for the sake of simplicity in this illustration. Also, two mesh resolutions are used in Fig. 4, a coarse one made of 100 cells (A) and a fine one made of 10,000 cells (B).

It appears that the speed of sound influences computed results. The wave speeds of the two-layer model (2.3) involve the effective sound speeds given by $\sqrt{c_{k}^{2}+\frac{1}{2} g h_{k}}$ while the singlelayer wave speed is $\sqrt{g h}$. When $c_{k}$ is significantly greater than $\sqrt{\frac{1}{2} g h_{k}}$ (of the order of unity in the present example), excessive numerical diffusion is present, as shown in Plot A of Fig. 4 where $c_{k}=100$ for both fluids. Indeed, at the current time, the left and right-facing waves are considerably dissipated and even exit the domain. However, this feature is purely numerical and the system does converge to the exact solution, as shown in Plot B of Fig. 4 that uses a fine grid made of 10, 000 cells. 

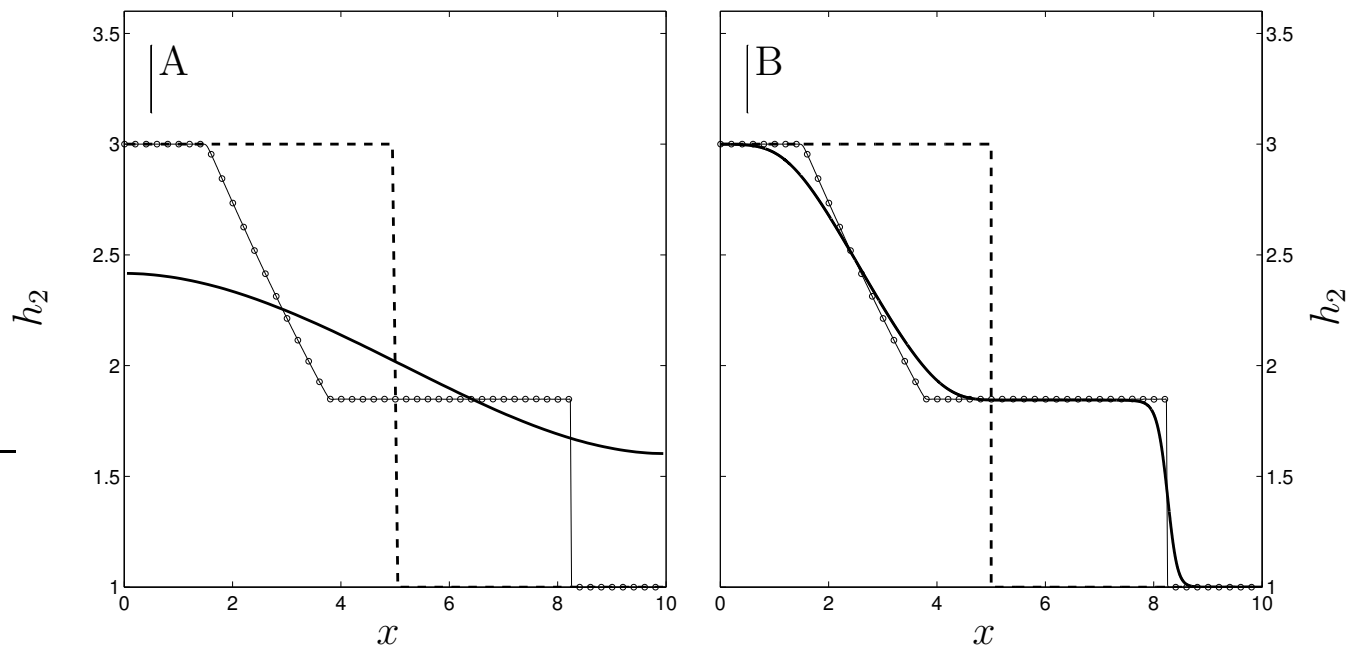

Figure 4: Comparison of the computed solutions with the present HLL-type Riemann solver (thick lines) versus the exact solution of the one-layer Saint-Venant equations (thin lines and symbols). Results on the left (A) use a 100-cell mesh while results on the right (B) use a 10,000-cell mesh. The two plots use constant sound speed set to $c_{k}=100$ for both fluids. The dashed lines represent the initial conditions. For the sake of clarity, 50 symbols are plotted for the exact solution, shown at time $t \approx 2$. First-order Godunov-type numerical scheme is used with $C F L=0.9$. Computed results are shown at the same time in full lines. Results at left (A) show a curved line where the shock and expansion waves have already exited the domain. Same computation is rerun with 10,000 cells (B) restoring the two waves inside the domain. All variables are in dimensionless units.

It thus appears that large sound speeds are admissible but result in excessive numerical diffusion. The effects of fluid compressibility and sound speed are then investigated by varying $c_{k}$ from levels less than the admissible single phase bound $\left(c_{k}<\sqrt{\frac{1}{2} g h_{k}}\right)$ to larger values. Corresponding results are shown in Fig. 5 ,

Numerical experiments of Fig. 5 indicate that the method becomes unstable when $c_{k}<$ $\sqrt{\frac{1}{2} g h_{k}}$. Indeed, Plot C of Fig. 5 uses $c_{k}=0.1 \times \sqrt{\frac{1}{2} g h_{k}}$ and presents spurious oscillations. These numerical experiments suggest existence of a subcharacteristic condition:

$$
c_{k}>\sqrt{\frac{1}{2} g h_{k}} .
$$

In the upcoming computations, the following sound speed is used:

$$
c_{k}=\theta_{k} \sqrt{\frac{1}{2} g h_{k}}, \quad \text { with } \quad \theta_{k}>1, \quad k=1,2 .
$$

$\theta_{k}$ is a numerical parameter that controls the numerical diffusion as illustrated in Fig. 5 , 

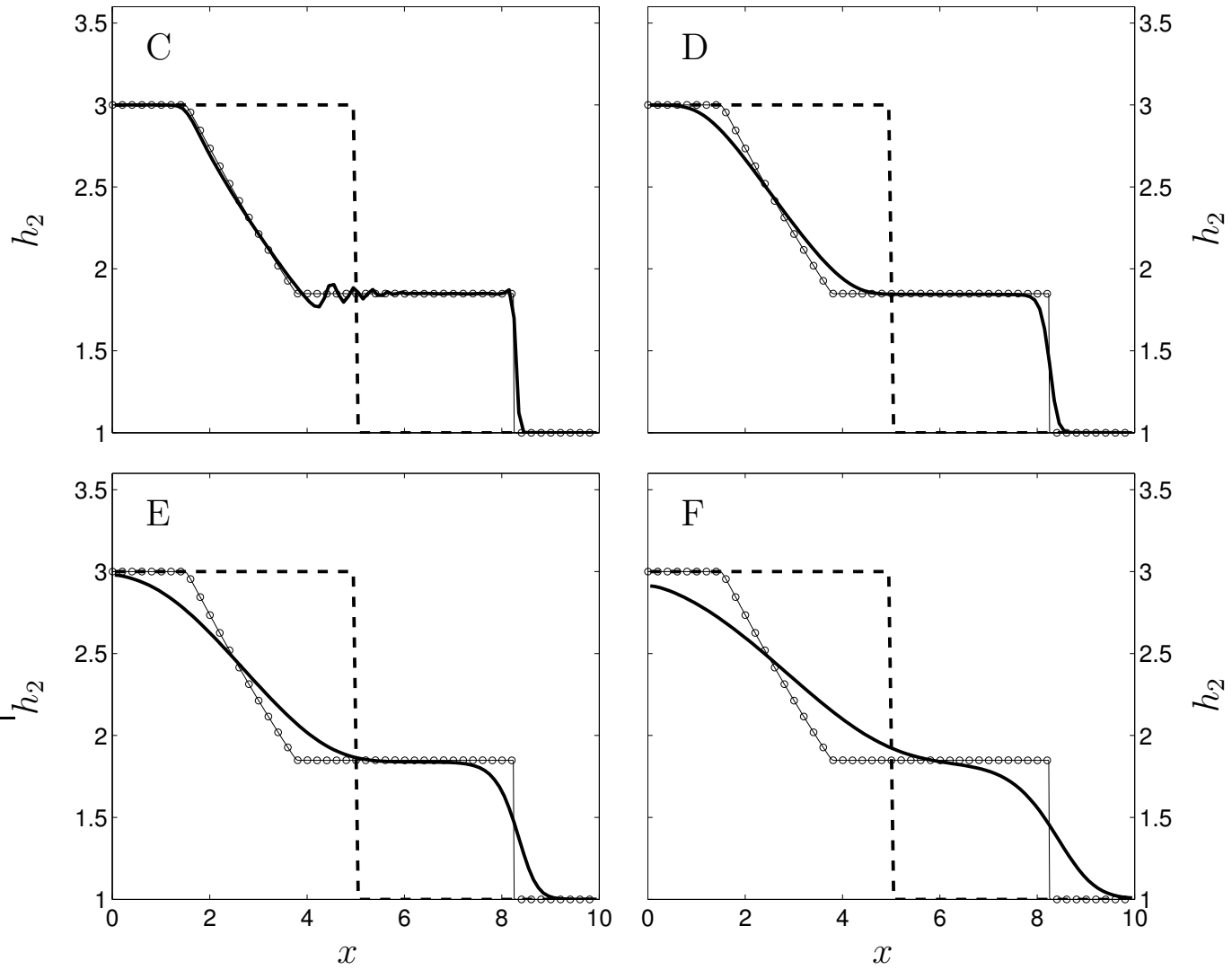

Figure 5: Influence of the artificial speed of sound. The computed solutions of the present HLL-type Riemann solver for the two-layer system are displayed in thick lines. The exact solution of the one-layer Saint-Venant equations is shown in thin lines and symbols. System (2.3) is solved with various sound speeds $c_{k}=\theta_{k} \sqrt{\frac{1}{2} g h_{k}}$. Figures $\mathrm{C}, \mathrm{D}, \mathrm{E}$ and $\mathrm{F}$ use respectively $\theta_{k}=0.1, \theta_{k}=2, \theta_{k}=10$ and $\theta_{k}=50$. The dashed lines represent the initial conditions: $h_{1}^{\text {left }}=h_{1}^{\text {right }}=10^{-6}, u_{1}^{\text {left }}=u_{1}^{\text {right }}=0, \rho_{1}=1.2, h_{2}^{\text {left }}=3, h_{2}^{\text {right }}=1, u_{2}^{\text {left }}=u_{2}^{\text {right }}=0$, $\rho_{2}=1$. Final time: $t \approx 2$. All results use a 100-cell mesh. First-order Godunov-type numerical scheme is used with $C F L=0.9$. For the sake of clarity, 50 symbols are plotted for the exact solution. An optimum appears for $\theta_{k}=2$. All variables are in dimensionless units. 
In order to unambiguously fulfill the above-mentioned subcharacteristic condition (4.1), $\theta_{k}$ must be greater than unit. $\theta_{k} \in[2,5]$ seems to be a fair choice as it is low enough to control numerical diffusion and high enough to ensure stability. Indeed, as seen in Plot D of Fig. 5, $\theta_{k}=2$ provides accurate results whereas $\theta_{k}=10$ (Plot $\mathrm{E}$ ) and $\theta_{k}=50$ (Plot $\mathrm{F}$ ) show excessive numerical dissipation.

Figure 6] repeats the same test with $\theta_{k}=2$ and a 1000-cell grid. The Godunov method (3.18) including non-conservation terms is extended to second order with the MUSCL-type method detailed for example in Toro (2013) [19] (see also Chiapolino et al. (2017) [21] when non-conservative terms are present).

The results show excellent agreement with the exact solution. Besides, they also reveal that:

- Incompressible behavior is recovered as the densities $\rho_{k}$ are constant;

- The two-layer shallow water model (2.3) tends to the single-layer Saint-Venant equations in the limit $h_{1} \rightarrow \epsilon$;

- Second-order extension of the Godunov-type scheme (3.18) and associated non-conservative terms does not pose specific difficulties.

These various computations have been done with the HLL solver while the VFRoe one failed immediately, as it was unable to preserve positivity of the height $h_{1}$. It is also important to note that the method does not require any fluid EOS, nor relaxation parameter, as Eq. (2.14) determines efficiently the heights at relaxed states. The only "thermodynamic" information is the sound speed, and more precisely $\theta_{k}$ in Eq. (4.2). The method is robust and accurate with $2 \leq \theta_{k} \leq 5$.

\subsection{Effects of the fluid densities}

The preceding dam-break problem showed that the two-layer shallow water model (2.3) is able to recover the single-layer Saint-Venant system in the limit $h_{1} \rightarrow 0$. When the density ratio $r=\frac{\rho_{2}}{\rho_{1}}$ is small, the effects of the surrounding fluid (upper layer) are expected to become insignificant and the one-layer Saint-Venant solution is meant to be recovered as well. 

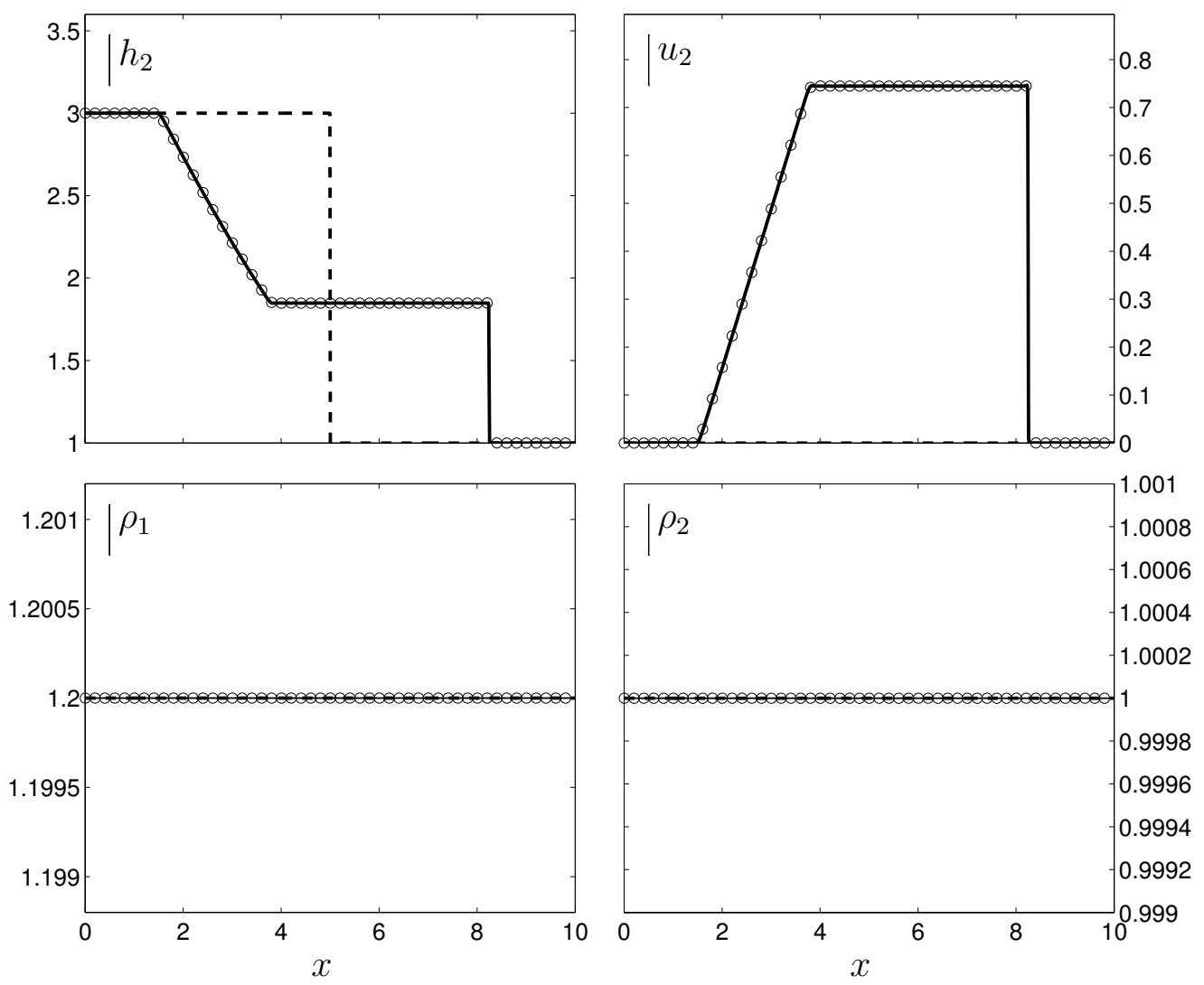

Figure 6: Comparison of the computed solutions with the present HLL-type Riemann solver (symbols) versus the exact solution of the one-layer Saint-Venant equations (thick lines). The numerical system uses an artificial sound speed reading $c_{k}=\theta_{k} \sqrt{\frac{1}{2} g h_{k}}$ with $\theta_{k}=2$. The dashed lines represent the initial conditions: $h_{1}^{\text {left }}=$ $h_{1}^{\text {right }}=10^{-6}, u_{1}^{\text {left }}=u_{1}^{\text {right }}=0, \rho_{1}=1.2, h_{2}^{\text {left }}=3, h_{2}^{\text {right }}=1, u_{2}^{\text {left }}=u_{2}^{\text {right }}=0, \rho_{2}=1$. Final time: $t \approx 2$. Second-order MUSCL-type numerical scheme using van Leer's limiter (see [22], [23]) is considered with $C F L=0.5$ and 1000 cells. For the sake of clarity, only 50 symbols out of 1000 are plotted for the HLL-type computations. All variables are in dimensionless units. 
The forthcoming tests analyze the effects of the fluid densities on a configuration presenting initially a Heaviside function regarding the height of the first fluid (lower layer) located in the middle of the numerical domain. The first layer is initially at height $h_{1}$ above the flat ground and the top of the plateau is located at height $h_{1}^{\prime}$. The second fluid surrounds the lower layer and is set initially at constant height $h_{2}$. The initial configuration is schematically depicted in Fig. 7 with data summarized in Table 1.

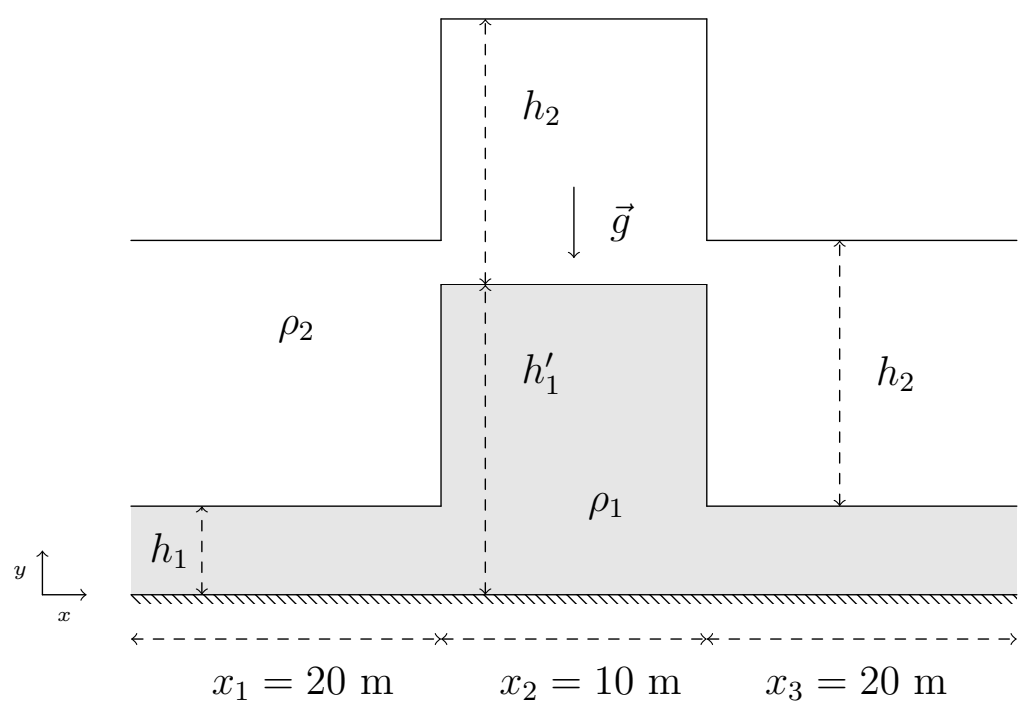

Figure 7: Schematic representation of the test problem analyzing the effects of the fluid densities. A fluid layer with a Heaviside profile is set to motion under gravity effects and interacts with the lighter fluid initially above with a Heaviside profile as well.

\begin{tabular}{cccccc}
\hline \hline Test & $h_{1}(\mathrm{~m})$ & $h_{1}^{\prime}(\mathrm{m})$ & $h_{2}(\mathrm{~m})$ & $\rho_{1}\left(\mathrm{~kg} \cdot \mathrm{m}^{-3}\right)$ & $\rho_{2}\left(\mathrm{~kg} \cdot \mathrm{m}^{-3}\right)$ \\
\hline $\mathrm{G}$ & 4 & 10 & 20 & 1000 & 1 \\
$\mathrm{H}$ & $10^{-6}$ & 10 & 20 & 1000 & 1 \\
$\mathrm{I}$ & 4 & 10 & 20 & 1000 & 990 \\
$\mathrm{~J}$ & $10^{-6}$ & 10 & 20 & 1000 & 990 \\
$\mathrm{~K}$ & 4 & 10 & 100 & 1000 & 990 \\
$\mathrm{~L}$ & $10^{-6}$ & 10 & 100 & 1000 & 990 \\
\hline \hline
\end{tabular}

Table 1: Initial conditions of the test problem analyzing the effects of the fluid densities.

In all following tests, the gravity constant is set to $g=10 \mathrm{~m} \cdot \mathrm{s}^{-2}$ and the top of the Heaviside plateau is at $h_{1}^{\prime}=10 \mathrm{~m}$. The results are shown at time $t=1 \mathrm{~s}$ and computed on a 1000-cell mesh with second-order MUSCL-type scheme and $C F L=0.5$. Figure 8 examines two different 
density ratios. Plots $\mathrm{G}$ and $\mathrm{H}$ consider $\rho_{1}=1000 \mathrm{~kg} \cdot \mathrm{m}^{-3}$ and $\rho_{2}=1 \mathrm{~kg} \cdot \mathrm{m}^{-3}$, this situation being typical of water-air configurations while Plots I, J, K and L consider $\rho_{1}=1000 \mathrm{~kg} \cdot \mathrm{m}^{-3}$ and $\rho_{2}=990 \mathrm{~kg} \cdot \mathrm{m}^{-3}$, a situation reminiscent of water-oil flows.

Besides, two different values of $h_{1}$ are used. The left column of Fig. 8 considers initially $h_{1}=4 \mathrm{~m}$ while the right column uses $h_{1}=10^{-6} \mathrm{~m}$. We will see that this initial data influences significantly computed results. All plots of Fig. 8 use $h_{2}=20 \mathrm{~m}$ with the exception of Plots K and $\mathrm{L}$ that use a much larger height for the upper layer, $h_{2}=100 \mathrm{~m}$. This data also influences the results.

Small density ratio: $r=\frac{\rho_{2}}{\rho_{1}} \ll 1$

When the density ratio is small, such as the situation of Fig. $8 \mathrm{G}$ and $\mathrm{H}$ considering a waterair-type configuration, the solutions of the two-layer system (2.3) are in excellent agreement with the exact solutions of the Saint-Venant model. For these two tests, $h_{1}^{\prime}=10 \mathrm{~m}, h_{2}=20 \mathrm{~m}$ and $h_{1}=4 \mathrm{~m}$ for Plot $\mathrm{G}$ and $h_{1}=10^{-6} \mathrm{~m}$ for Plot $\mathrm{H}$.

In Plot G of Fig. 8, the Heaviside profile of the lower layer gives rise to four waves moving in each direction. The extreme waves steepen through compression waves into two shocks, while the back waves spread out as rarefaction waves.

However, the solution in Plot $\mathrm{H}$ of Fig. 8 is significantly different as the initial height of the lower layer is as low as numerically acceptable (outside the Heaviside profile). Thereby the solution evolves continuously throughout the entire numerical domain as there is not enough fluid, regarding the lower layer $\left(h_{1}\right)$, to observe a compression process: expansion waves only are present.

Comparable densities: $r=\frac{\rho_{2}}{\rho_{1}} \rightarrow 1$

Plots I and J of Fig. 8 repeat the same test $\left(h_{1}^{\prime}=10 \mathrm{~m}, h_{2}=20 \mathrm{~m}, h_{1}=4 \mathrm{~m}\right.$ (Plot I) and $h_{1}=10^{-6} \mathrm{~m}($ Plot J)) but with a water-oil-type configuration. As expected, the solutions are different from the one-fluid solutions, as the two fluids are dense and interact each other, this interaction being taken into account by the two-fluid model only. The two-layer solutions are shown with full lines and the single-layer Saint-Venant solution is shown with symbols, just to compare the limit solutions. 

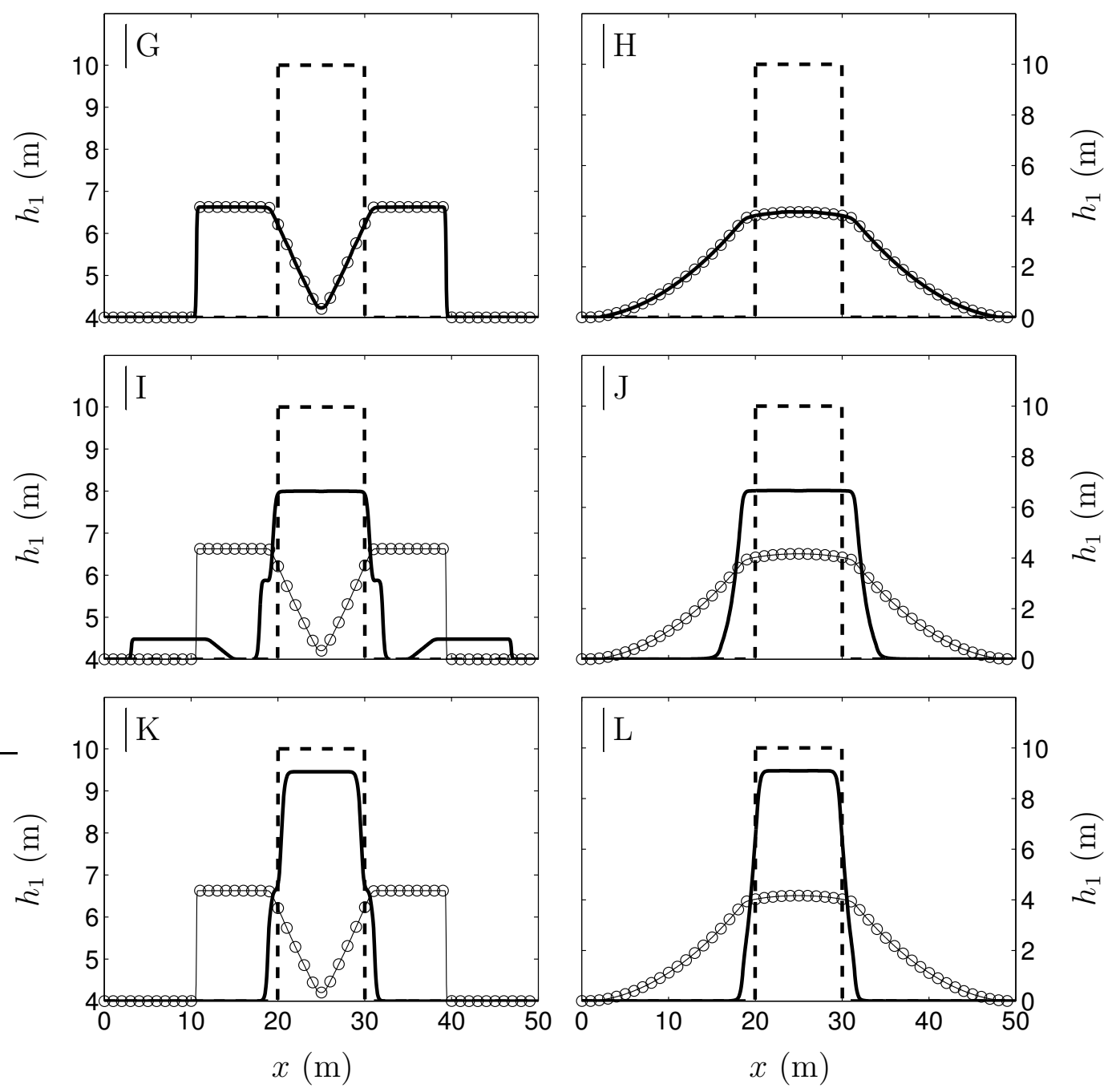

Figure 8: Influence of the density ratio between the two fluids. Two different density ratios are used: $r=\frac{\rho_{2}}{\rho_{1}} \ll 1$ and $r=\frac{\rho_{2}}{\rho_{1}} \rightarrow 1$. The computed solutions of the present HLL-type Riemann solver for the two-layer system are displayed in thick lines. The exact solutions of the one-layer Saint-Venant equations are shown in thin lines and symbols for comparison. System (2.3) uses an artificial sound speed reading $c_{k}=\theta_{k} \sqrt{\frac{1}{2} g h_{k}}$ with $\theta_{k}=2$. The dashed lines represent the initial conditions. Final time: $t \approx 1 \mathrm{~s}$. All results use a 1000-cell mesh. Second-order MUSCL-type numerical scheme is used with Sweby's limiter $(\phi=1.1$, see [23]) and $C F L=0.5$. For the sake of clarity, 50 symbols are plotted for the exact solution of the single-layer model. On the column at left, the initial height is $h_{1}=4 \mathrm{~m}$ and on the column at right $h_{1}=10^{-6} \mathrm{~m}$. When the density ratio is small: $r=\frac{\rho_{2}}{\rho_{1}} \ll 1$, as in configurations $\mathrm{G}$ and $\mathrm{H}$, the two-layer and single-layer models are in perfect agreement. Large differences appear when the density ratio increases as shown in configurations I, J, K and L. 
The interaction of the two fluids influences significantly the computed results as an interesting wave structure appears in Plot I of Fig. 8. Right and left-facing shock waves propagate faster than those of the single-layer system. These shocks induce height increase of the fluid layer. They are followed by expansion waves that decrease these heights. Contact waves follow these expansion waves, followed by extra expansion waves that decrease the initial height $h_{1}^{\prime}$.

The solution is quite different when $h_{1}=10^{-6} \mathrm{~m}$ initially (Plot $\mathrm{J}$ ) where only two expansion waves are observable. The interaction of the two fluids influences the flow, as the first fluid moves more difficultly into the second one as a result of comparable densities.

Large upper layer

Plots K and L of Fig. 8 keep on analyzing the present density ratio (water-oil) but with different height for the second fluid that is now set to $h_{2}=100 \mathrm{~m}$. Doing so, the domain is mainly filled with the upper layer and Plots $\mathrm{K}$ and $\mathrm{L}$ show that the first fluid moves into the second one difficultly. The difference of initial height for the first fluid $h_{1}=4 \mathrm{~m}(\mathrm{Plot} \mathrm{K})$ and $h_{1}=10^{-6} \mathrm{~m}$ (Plot L) is minor compared to the effect of the large layer of second fluid $\left(h_{2}=100\right.$ $\mathrm{m})$. As the entire domain is mainly filled with heavy fluids, the flow is slowly set to motion under gravity effects.

\section{Concluding remarks}

Those last results reveal that the two-fluid model (2.3) is able to recover the one-layer SaintVenant system when the effects of the surrounding fluid are negligible, as expected. This behavior appears when the density ratio between the lightest fluid and the heaviest one is small: $r=$ $\frac{\rho_{2}}{\rho_{1}} \ll 1$.

They also reveal the importance of the two-layer model when the density ratio is arbitrary. Indeed, the two-layer system is able to deal with interactions between fluids unlike the conventional one-fluid Saint-Venant model. Note that the previous tests have been computed with the HLL-type Riemann solver presented in Section [3.2, the VFRoe method being unable to keep positivity of the heights. Note also that drag effects are absent in these computations. 


\subsection{Comparison of VFRoe and HLL}

The two solvers considered in the present paper are tested on a flow configuration examined in Abgrall and Karni (2009) [10] and Kurganov and Petrova (2009) [13]. The following tests set gravity constant to $g=10 \mathrm{~m} . \mathrm{s}^{-2}$ and density ratio to $r=\frac{\rho_{2}}{\rho_{1}}=0.98$. Hereby, $\rho_{1}=1200 \mathrm{~kg} \cdot \mathrm{m}^{-3}$ and $\rho_{2}=1176 \mathrm{~kg} \cdot \mathrm{m}^{-3}$ are used. Initially, different heights are present from either side of the initial discontinuity and results in the creation of a flow under gravity effects. The numerical domain is $1 \mathrm{~m}$ long and the initial discontinuity is located at $x=0.5 \mathrm{~m}$. On the left of this discontinuity $h_{1}=0.5 \mathrm{~m}$ and $h_{2}=0.5 \mathrm{~m}$. On the right, $h_{1}=0.45 \mathrm{~m}$ and $h_{2}=0.55 \mathrm{~m}$. The initial conditions are schematically depicted in Fig. 9. The first-order Godunov-type scheme is used with $C F L=0.7$ in the following tests. Doing so, the comparison between solvers is free of extra ingredients, such as gradient limiters.

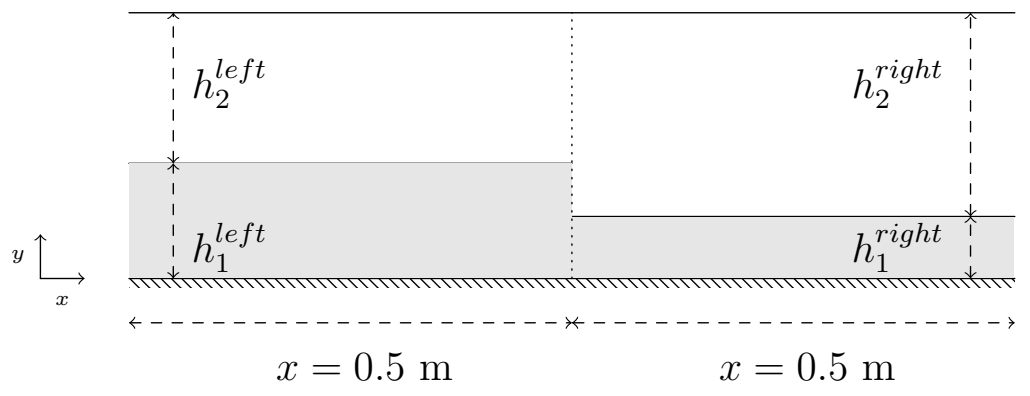

Figure 9: Schematic representation of the test problem comparing the computed solutions with the present HLLtype Riemann solver and those computed with the VFRoe method. The initial conditions are $h_{1}^{\text {left }}=0.5 \mathrm{~m}$, $h_{1}^{\text {right }}=0.45 \mathrm{~m}, h_{2}^{\text {left }}=0.5 \mathrm{~m}, h_{2}^{\text {right }}=0.55 \mathrm{~m}, \rho_{1}=1200 \mathrm{~kg} \cdot \mathrm{m}^{-3}, \rho_{2}=1176 \mathrm{~kg} \cdot \mathrm{m}^{-3}$. The initial velocities are: $u_{1}^{\text {left }}=u_{1}^{\text {right }}=u_{2}^{\text {left }}=u_{2}^{\text {right }}=0 \mathrm{~m} \cdot \mathrm{s}^{-1}$ in Figs. 10 and 11 and $u_{1}^{\text {left }}=u_{1}^{\text {right }}=u_{2}^{\text {left }}=u_{2}^{\text {right }}=2.5 \mathrm{~m} . \mathrm{s}^{-1}$ in Figs. 12 and 13

Figure 10 displays the results obtained with the HLL-type solver and the VFRoe method on a 100-cell mesh. The initial velocity is set to $u_{1}=u_{2}=0 \mathrm{~m} \cdot \mathrm{s}^{-1}$ throughout the entire domain. The results are shown at time $t \approx 0.12 \mathrm{~s}$.

The hyperbolic model (2.3) considers compressible fluids during the wave propagation stage. However, the pressure relaxation step restores incompressibility as shown in Fig. 10 where the densities $\rho_{1}$ and $\rho_{2}$ remain constant. The numerical solutions consist in 4 waves emerging from the initial discontinuity. Both methods predict the same solution qualitatively, but the VFRoe solver produces spurious oscillations while the HLL one is monotonic. 

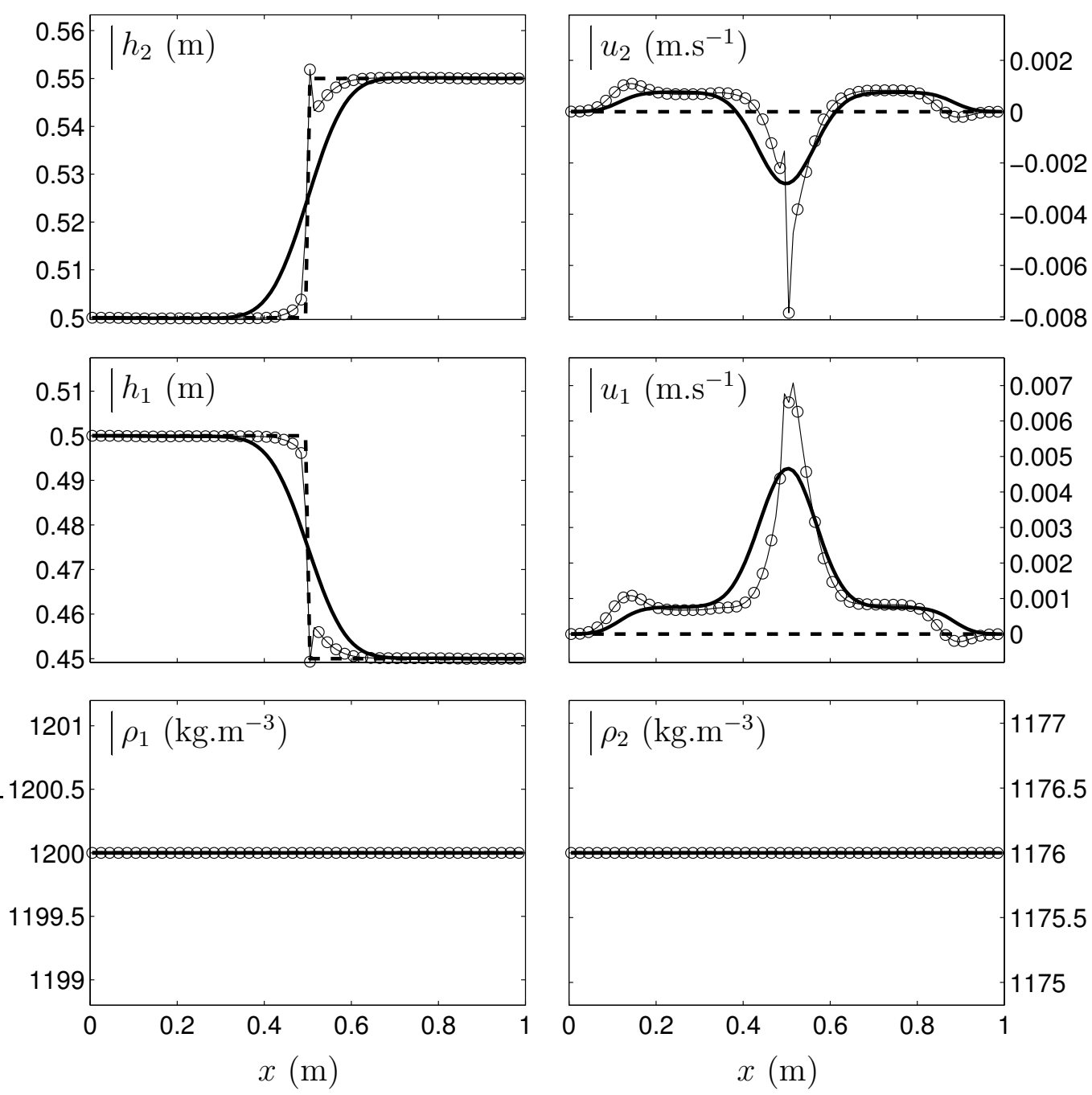

Figure 10: Comparison of the computed solutions with the present HLL-type Riemann solver (thick lines) versus the computed solutions with the VFRoe method (thin lines and symbols). The dashed lines represent the initial conditions: $h_{1}^{\text {left }}=0.5 \mathrm{~m}, h_{1}^{\text {right }}=0.45 \mathrm{~m}, h_{2}^{\text {left }}=0.5 \mathrm{~m}, h_{2}^{\text {right }}=0.55 \mathrm{~m}, u_{1}^{\text {left }}=u_{1}^{\text {right }}=u_{2}^{\text {left }}=u_{2}^{\text {right }}=0$ $\mathrm{m} . \mathrm{s}^{-1}, \rho_{1}=1200 \mathrm{~kg} \cdot \mathrm{m}^{-3}, \rho_{2}=1176 \mathrm{~kg} . \mathrm{m}^{-3}$. For the first fluid, the numerical parameter reads $\theta_{1}=3.5$, for the second fluid, $\theta_{2}=3$. These parameters are minimum values for successful computations with VFRoe. Final time: $t \approx 0.12 \mathrm{~s}$. First-order Godunov-type numerical scheme is used with $C F L=0.7$ and 100 cells. For the sake of clarity, only 50 symbols out of 100 are plotted for the VFRoe method. 
As initially $u_{1}^{\text {left }}=u_{1}^{\text {right }}=u_{2}^{\text {left }}=u_{2}^{\text {right }}=0 \mathrm{~m} \cdot \mathrm{s}^{-1}$, the velocity $\bar{u}=0 \mathrm{~m} \cdot \mathrm{s}^{-1}$ and the full state vector $\bar{W}$ in the VFRoe solver induces singularities in the decomposition of the eigenvectors. To lower this effect, different sound speeds (through $\theta_{1}$ and $\theta_{2}$ ) have been considered in the various fluids. In the absence of such correction, the VFRoe solver fails immediately. Consequently, $\theta_{1} \neq \theta_{2}$ is used as well with the HLL-type solver for proper comparison.

Figure [1] addresses the same test problem on a 10,000-cell mesh, showing convergence to the same solution of both VFRoe and HLL. With refined mesh, the interfacial waves are clearly connected to a constant plateau spanning as time evolves. Besides the four-wave structure is clearly observable in the velocity plots.
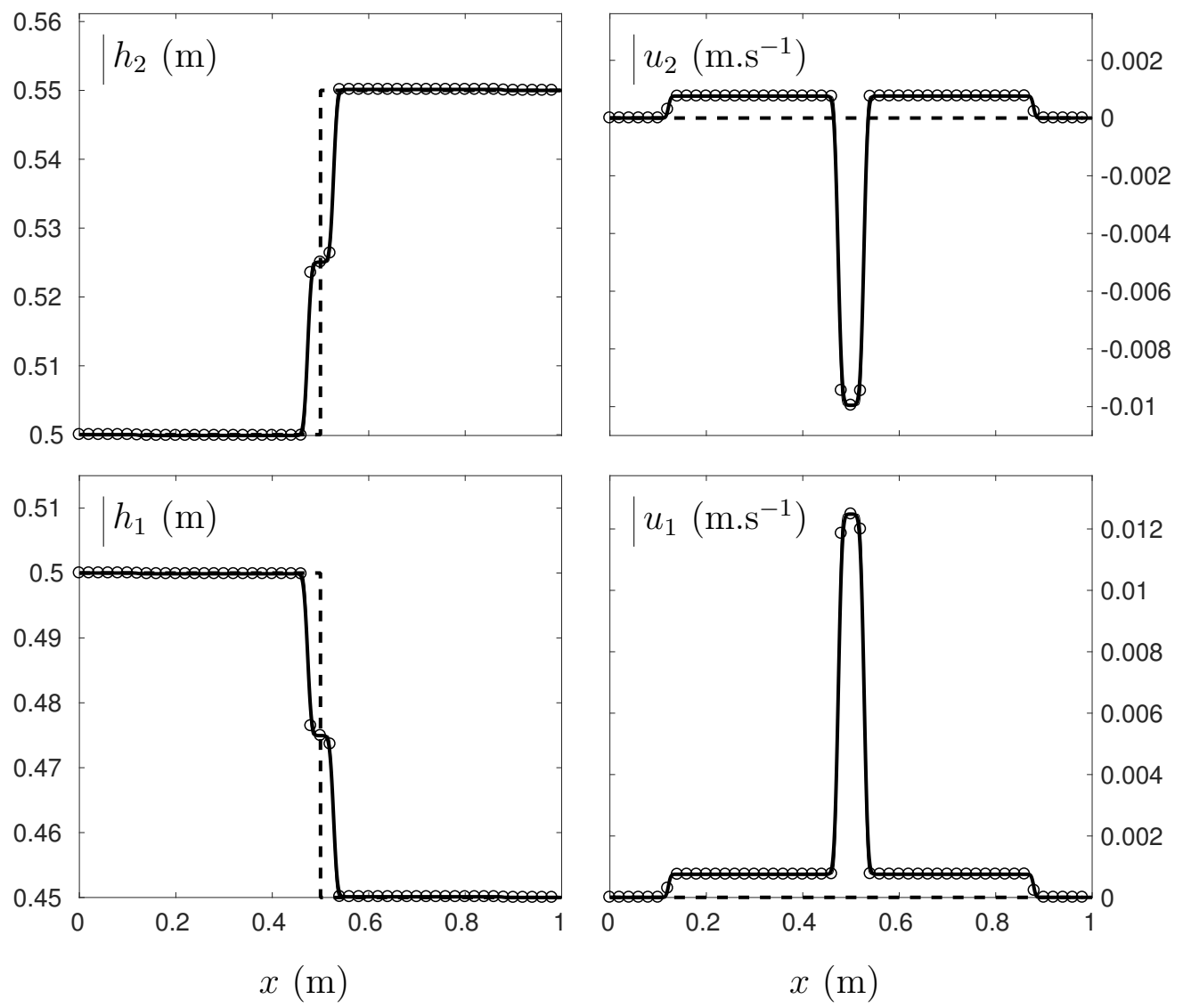

Figure 11: Comparison of the computed solutions with the present HLL-type Riemann solver (thick lines) versus the computed solutions with the VFRoe method (thin lines and symbols). The dashed lines represent the initial conditions: $h_{1}^{\text {left }}=0.5 \mathrm{~m}, h_{1}^{\text {right }}=0.45 \mathrm{~m}, h_{2}^{\text {left }}=0.5 \mathrm{~m}, h_{2}^{\text {right }}=0.55 \mathrm{~m}, u_{1}^{\text {left }}=u_{1}^{\text {right }}=u_{2}^{\text {left }}=u_{2}^{\text {right }}=0$ $\mathrm{m} . \mathrm{s}^{-1}, \rho_{1}=1200 \mathrm{~kg} . \mathrm{m}^{-3}, \rho_{2}=1176 \mathrm{~kg} . \mathrm{m}^{-3}$. For the first fluid, the numerical parameter reads $\theta_{1}=3.5$, for the second fluid, $\theta_{2}=3$. Final time: $t \approx 0.12 \mathrm{~s}$. First-order Godunov-type numerical scheme is used with $C F L=0.7$ and 10,000 cells. For the sake of clarity, only 50 symbols out of 10,000 are plotted for the VFRoe method. Both methods converge to the same solution. 
The next test repeats the previous one with non-zero initial velocities. Those are set to $u_{1}=u_{2}=2.5 \mathrm{~m} . \mathrm{s}^{-1}$ throughout the entire domain. This test was examined in Abgrall and Karni (2009) [10] and computed with both 400 and 10,000-cell meshes. The same grids are used here to compare the present model and HLL solver with the results given in [10]. Figure 12 shows the results at time $t \approx 0.07 \mathrm{~s}$ with 400 -cell mesh.
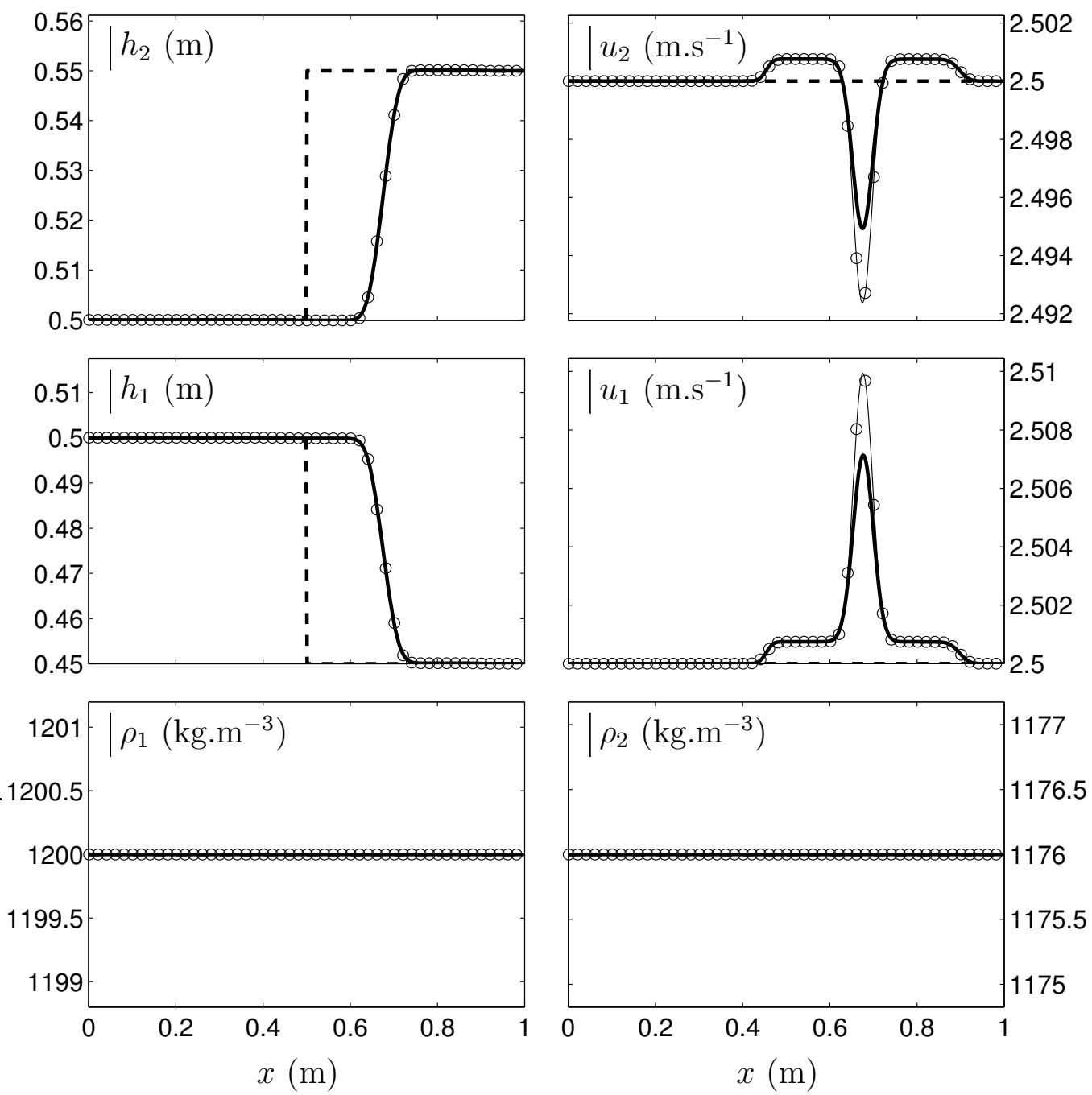

Figure 12: Comparison of the computed solutions with the present HLL-type Riemann solver (thick lines) versus the computed solutions with the VFRoe algorithm (thin lines and symbols). The dashed lines represent the initial conditions: $h_{1}^{\text {left }}=0.5 \mathrm{~m}, h_{1}^{\text {right }}=0.45 \mathrm{~m}, h_{2}^{\text {left }}=0.5 \mathrm{~m}, h_{2}^{\text {right }}=0.55 \mathrm{~m}, u_{1}^{\text {left }}=u_{1}^{\text {right }}=u_{2}^{\text {left }}=u_{2}^{\text {right }}=2.5$ $\mathrm{m} . \mathrm{s}^{-1}, \rho_{1}=1200 \mathrm{~kg} . \mathrm{m}^{-3}, \rho_{2}=1176 \mathrm{~kg} . \mathrm{m}^{-3}$. For the first fluid, the numerical parameter reads $\theta_{1}=3.5$, for the second fluid, $\theta_{2}=3$. Final time: $t \approx 0.07 \mathrm{~s}$. First-order Godunov-type numerical scheme is used with $C F L=0.7$ and 400 cells. For the sake of clarity, only 50 symbols out of 400 are plotted for the VFRoe method.

As a consequence of non-zero initial velocities and fine mesh resolution, computational con- 
ditions are easier for the VFRoe-type solver that does not oscillate. The expected behavior is recovered. The heights and velocity profiles are transported to the right and the effects of gravity seen in Figs. 10 and 11 are still present. As expected the densities remain constant as a consequence of pressure relaxation. Figure 13 shows the results of the same test with 10,000-cell mesh.

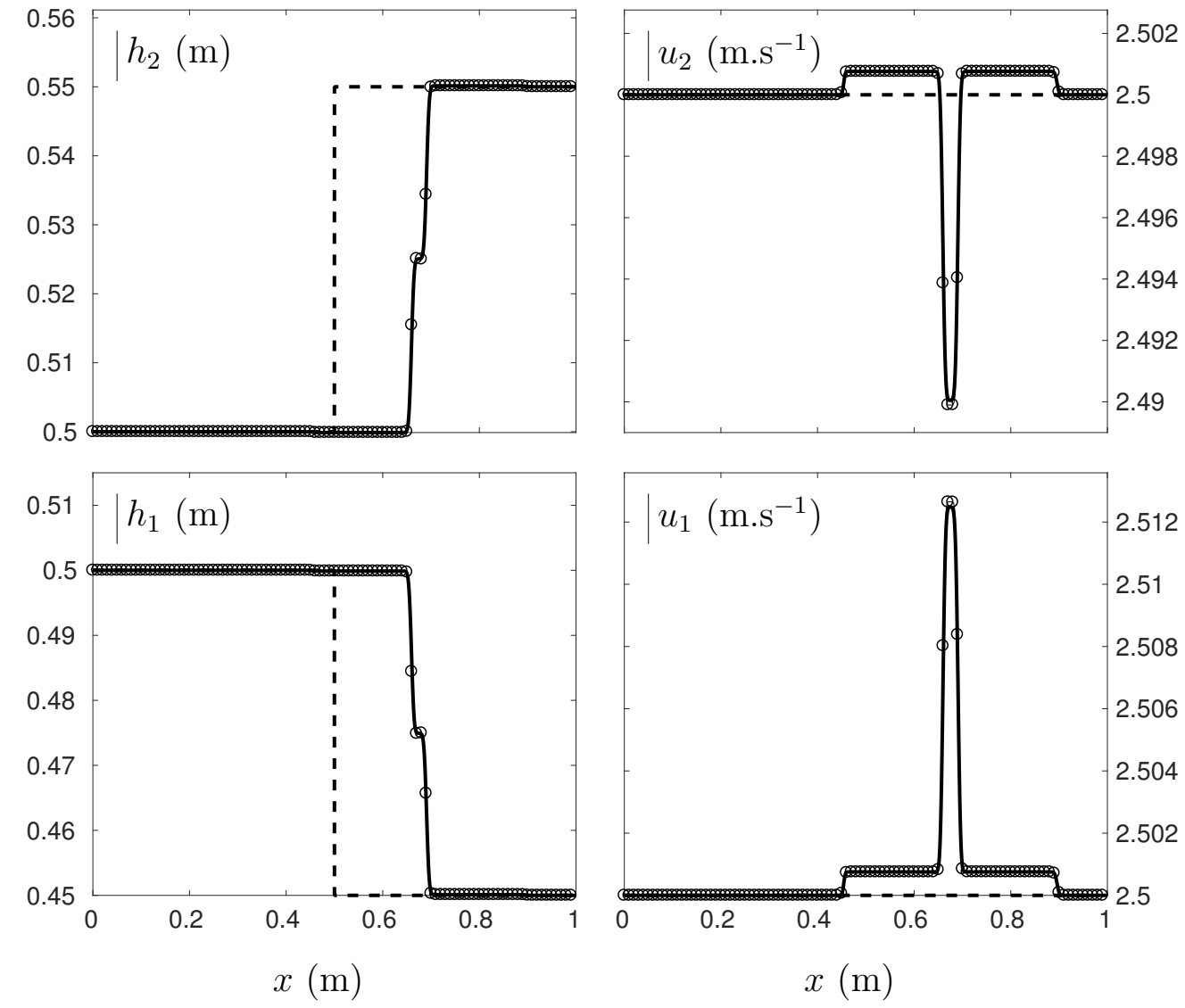

Figure 13: Comparison of the computed solutions with the present HLL-type Riemann solver (thick lines) versus the computed solutions with the VFRoe algorithm (thin lines and symbols). The dashed lines represent the initial conditions: $h_{1}^{\text {left }}=0.5 \mathrm{~m}, h_{1}^{\text {right }}=0.45 \mathrm{~m}, h_{2}^{\text {left }}=0.5 \mathrm{~m}, h_{2}^{\text {right }}=0.55 \mathrm{~m}, u_{1}^{\text {left }}=u_{1}^{\text {right }}=u_{2}^{\text {left }}=u_{2}^{\text {right }}=2.5$ $\mathrm{m} . \mathrm{s}^{-1}, \rho_{1}=1200 \mathrm{~kg} . \mathrm{m}^{-3}, \rho_{2}=1176 \mathrm{~kg} . \mathrm{m}^{-3}$. For the first fluid, the numerical parameter reads $\theta_{1}=3.5$, for the second fluid, $\theta_{2}=3$. Final time: $t \approx 0.07 \mathrm{~s}$. First-order Godunov-type numerical scheme is used with $C F L=0.7$ and 10,000 cells. For the sake of clarity, only 100 symbols out of 10,000 are plotted for the VFRoe method.

The HLL-type solver and the VFRoe-type method are in excellent agreement and are in excellent agreement with the results given in Abgrall and Karni (2009) [10] as well.

It is interesting to examine the rate of convergence of the present method (HLL-type solver) and give comparison to existing methods. In Abgrall and Karni (2009) [10], only first-order 
computations were carried out while in Kurganov and Petrova (2009) [13], higher-order computational results are provided. Comparisons are consequently done with the results of figures 2.2, 2.3 and 2.4 of this last reference. The results are given in Fig. 14,

Figure 14 shows results comparable to those of Kurganov and Petrova (2009) [13] with all mesh sizes. The second-order MUSCL-type scheme provides velocity profiles accurate enough with both 400-cell and 800-cell meshes as observed in [13]. However, the velocity spike with the 800-cell mesh seems slightly greater than the reference solution and the close-up view on the height profile reveals that only the 800-cell mesh cancels out sufficiently numerical dissipation and allows to observe properly the constant plateau. The same conclusions are obtained in Kurganov and Petrova (2009) [13].

Also, a slight oscillation is observed with the coarse mesh made of 100 cells. The same observation holds in [13]. However, the left and right-facing shocks, seen on the velocity profile,

differ from those observed in [13] where initial data seem to be not exactly the same as those of Abgrall and Karni (2009) [10]. The present results are nonetheless in agreement with the ones of Abgrall and Karni (2009) [10]. The overall height is consequently different from the results of Kurganov and Petrova (2009) [13]. Nevertheless, velocity profiles indicate that the present method seems to have a similar rate of convergence as the one of [13].

Note that for this test problem, $\theta_{1}=\theta_{2}=2$ induces spurious oscillations, similar to those observed in Fig. 5, Plot C. Consequently, $\theta_{1}=\theta_{2}=3$ is used for both fluids.

\section{Comparison of two-layer shallow water solutions versus two-dimensional two-fluid computations}

The averaged (or homogenized) solution computed by the present one-dimensional two-layer shallow water system (2.3) is now compared to the solution of a multidimensional model involving material interfaces. As mentioned in the introduction, the two-layer approach is expected to provide comparable results with considerable computational savings.

In the following, solutions computed with the compressible two-phase flow model of Saurel et al. (2009) [24] are used as reference solutions. This model is a pressure disequilibrium system which tends, in its asymptotic limit of stiff pressure relaxation, to the model of Kapila et al. 

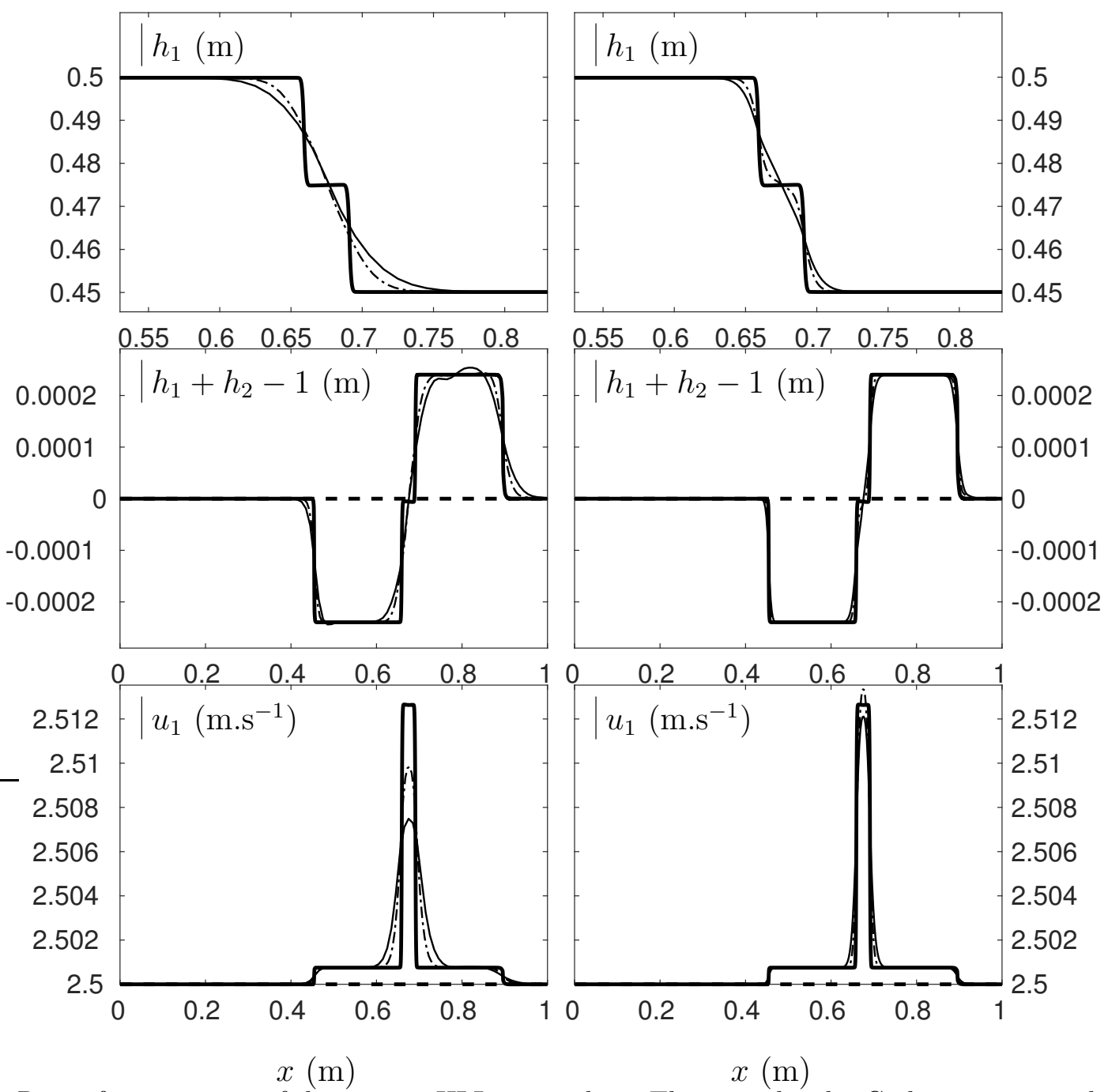

Figure 14: Rate of convergence of the present HLL-type solver. The second-order Godunov-type method is used with Minmod limiter and with four mesh resolutions. The thin solid lines represent results obtained with 100 cells (left column) and 400 cells (right column). The dashed-dotted lines represent results obtained with 200 cells (left column) and 800 cells (right column). The thick solid lines represent the results obtained with 10,000 cells and considered as "reference" solution. The thick dashed lines represent the initial conditions: $h_{1}^{\text {left }}=0.5 \mathrm{~m}$, $h_{1}^{\text {right }}=0.45 \mathrm{~m}, h_{2}^{\text {left }}=0.5 \mathrm{~m}, h_{2}^{\text {right }}=0.55 \mathrm{~m}, u_{1}^{\text {left }}=u_{1}^{\text {right }}=u_{2}^{\text {left }}=u_{2}^{\text {right }}=2.5 \mathrm{~m} . \mathrm{s}^{-1}, \rho_{1}=1200 \mathrm{~kg} \cdot \mathrm{m}^{-3}$, $\rho_{2}=1176 \mathrm{~kg} \cdot \mathrm{m}^{-3}$. For the two fluids, the numerical parameters read $\theta_{1}=\theta_{2}=3$. Final time: $t \approx 0.07 \mathrm{~s}$. The CFL number is 0.7. A close-up view of the spanning plateau is provided at top with the $h_{1}$ profile. The overall height $h_{1}+h_{2}+B(x)$ is given in the middle. $B(x)$ represents topography of the ground and is considered constant in the present work, $B(x)=0 \mathrm{~m}$. For proper comparison with the results of Kurganov and Petrova (2009) 13. who considered $B(x)=-1 \mathrm{~m}$, unit is subtracted from $h_{1}+h_{2}$. Finally the velocity profile $u_{1}$ is displayed at bottom. 
(2001) [15], able to compute fluid interfaces as diffuse numerical zones. Interface sharpening can be achieved with the method of Chiapolino et al. (2017) [21].

To compare the solutions computed by both approaches (multidimensional interface model and one-dimensional two-layer shallow water one), the test configuration schematically depicted in Fig. 15 is used with data summarized in Table 2.

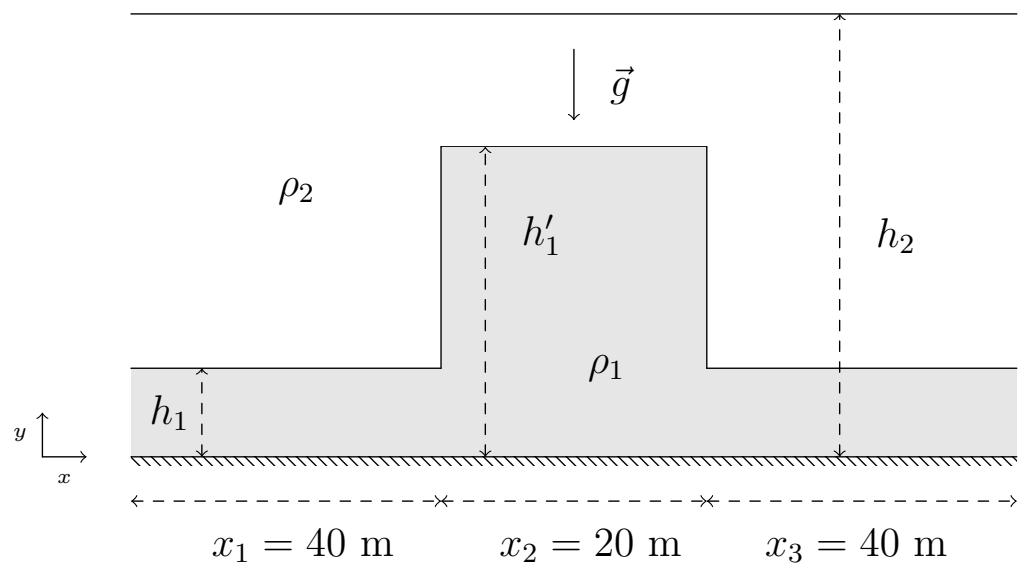

Figure 15: Schematic representation of the test problem comparing the multidimensional interface approach and the one-dimensional two-layer shallow water model. A fluid layer with a Heaviside profile is set to motion under gravity effects and interacts with the lighter fluid initially above.

\begin{tabular}{cccccccc}
\hline \hline Test & $h_{1}(\mathrm{~m})$ & $h_{1}^{\prime}(\mathrm{m})$ & $h_{2}(\mathrm{~m})$ & $\rho_{1}\left(\mathrm{~kg} \cdot \mathrm{m}^{-3}\right)$ & $\rho_{2}\left(\mathrm{~kg} . \mathrm{m}^{-3}\right)$ & $\gamma_{1}$ & $\gamma_{2}$ \\
\hline $1 \mathrm{D} / 2 \mathrm{D}$ & $10^{-6}$ & 1 & 10 & 3.506 & 1.29 & 1.67 & 1.4 \\
\hline \hline
\end{tabular}

Table 2: Initial conditions of the test problem comparing the multidimensional interface approach and the onedimensional two-layer shallow water model. $\gamma_{k}$ represents the thermodynamic polytropic coefficient of fluid $k$ used in the compressible two-phase flow model.

Krypton and air are considered at rest and at atmospheric conditions initially. The flow is set to motion by the gravity acceleration $|g|=10 \mathrm{~m} \cdot \mathrm{s}^{-2}$ and consists of two ideal gases evolving at low Mach number. Due to symmetric boundary conditions, only half of the domain is computed with the multidimensional approach. This latter uses an unstructured mesh made of 510,000 triangular elements with spatial discretization varying from about $1.5 \mathrm{~cm}$ in the zone of interest

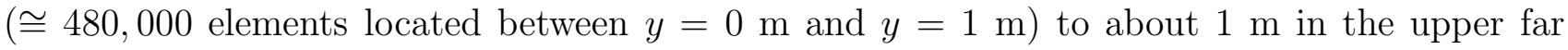
field. The numerical boundaries are considered as atmospheric outflows except for the left side (symmetric condition) and bottom one (flat ground) considered as walls. 
The accurate resolution of interfaces in multidimensional computations is improved with a second-order numerical method. The MUSCL-type method with "Overbee" limiter at interfaces is used as detailed in [21]. The very same second-order method is used with the two-layer shallow water system, computed on a 1000-cell mesh with van Leer's limiter [22]. Non-reflecting boundary conditions are used for the shallow water computations.

Results are shown in Fig. 16 at times $t=5 \mathrm{~s}$ and $t=8 \mathrm{~s}$. The computed averaged height and averaged velocity of the multidimensional computation are determined by the integration on the two-dimensional numerical domain as,

$$
h_{1}=\int_{0}^{h_{2}} \alpha_{1} d y
$$

and

$$
u_{x}=\frac{\int_{0}^{h_{2}}\left(\alpha_{1} \rho_{1} u_{x}\right) d y}{\int_{0}^{h_{2}}\left(\alpha_{1} \rho_{1}\right) d y},
$$

where $\alpha_{1}$ denotes the volume fraction of krypton and $u_{x}$ denotes the averaged velocity of the two-phase mixture in the $x$-axis direction. The one-fluid shallow water solution is shown as well in Fig. 16 for comparison.

As expected, the two-layer model provides better results than the one-fluid model. The density ratio $r=\frac{\rho_{2}}{\rho_{1}}=\frac{1.29}{3.506} \simeq 0.37$ being moderate, the interaction between the two fluids is meant to be significant. The results of the two-layer shallow water system present a large zone where the agreement with the two-dimensional simulation is very good. Beyond this zone, the results computed by the multidimensional interface model present oscillations. These oscillations are due to the presence of Kelvin-Helmholtz instabilities as seen for example in Fig. 17 showing the $2 \mathrm{D}$ results at time $t=2 \mathrm{~s}$.

Indeed, the multidimensional solution involves hydrodynamic instabilities that cannot be accounted for with the present two-layer shallow water model. The overall qualitative behavior of the one-dimensional approach is correct but the krypton is spread too far ahead with the two-layer model.

To improve agreement between 1D and 2D computations, drag effects are added in the two- 

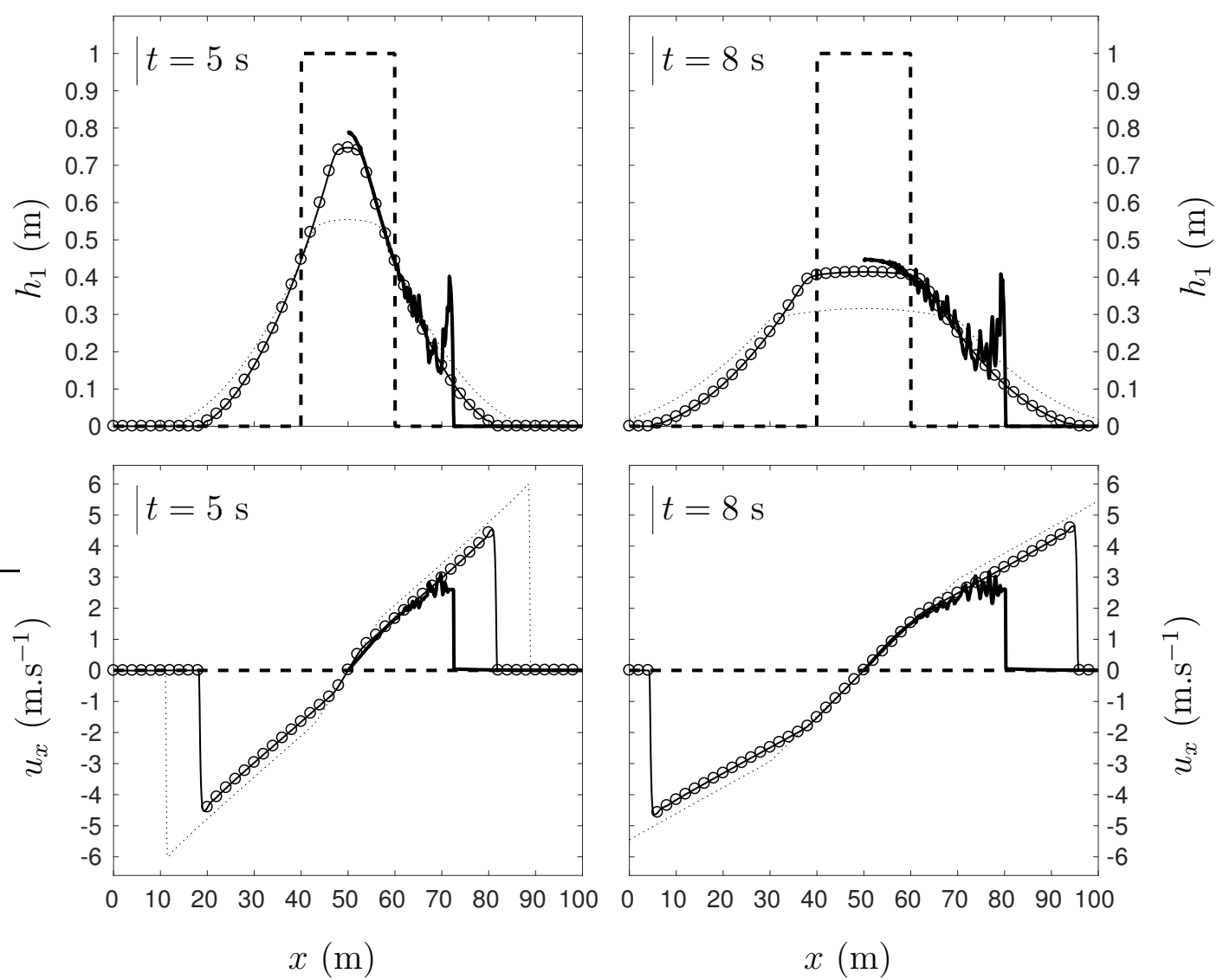

Figure 16: Comparison of the one-dimensional solution (thin lines and symbols) of the present two-layer shallow water model (HLL-type solver, $\theta_{k}=2$ ) versus the two-dimensional computation of the diffuse interface model of Saurel et al. (2009) 24] (thick lines). The exact solution of the one-layer Saint-Venant equations is plotted (dotted lines) as well for comparison. The dashed lines represent the initial conditions. For the sake of clarity, 50 symbols are plotted for the two-layer solution. The column at left shows the results at time $t=5 \mathrm{~s}$ and the column at right shows the same results at time $t=8 \mathrm{~s}$. For symmetry reasons, only half of the numerical domain is computed with the two-dimensional simulation. Both computations (two-layer and diffuse interface systems) use $C F L=0.8$.

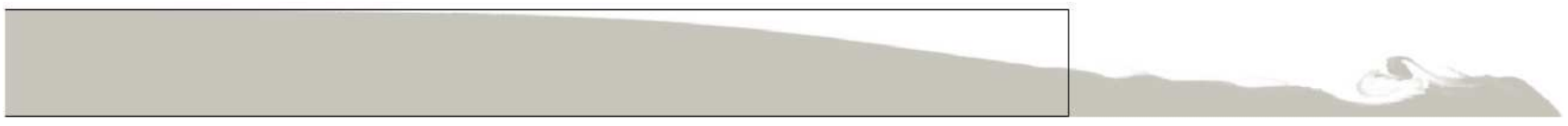

Figure 17: Kelvin-Helmholtz instabilities observed during the descent of krypton due to gravity effects, $g=-10$ $\mathrm{m} . \mathrm{s}^{-2}$. The figure presents krypton volume fraction contours. The black rectangle represents the initial position of the gas. The results are shown at time $t=2 \mathrm{~s}$ and are computed with the diffuse interface model of Saurel et al. (2009) 24] on an unstructured mesh made of 510,000 triangular elements. MUSCL-type method is used with the "sharpening-interface" method of Chiapolino et al. (2017) 21] and $C F L=0.8$. For symmetry reasons, only half of the numerical domain is computed. 
layer formulation. Pressure (or "acoustic") drag is considered only and is modeled through the velocity relaxation terms that appear in the right-hand side of the momentum equations,

$$
\left\{\begin{array}{l}
\frac{\partial\left(h_{1} \rho_{1} u_{1}\right)}{\partial t}+\frac{\partial\left(h_{1} \rho_{1} u_{1}^{2}+h_{1} p_{1}\left(\rho_{1}\right)+\frac{1}{2} \rho_{1} g h_{1}^{2}+g \rho_{2} h_{1} h_{2}\right)}{\partial x}=\rho_{2} g h_{2} \frac{\partial h_{1}}{\partial x}+p_{0} \frac{\partial h_{1}}{\partial x}+\frac{Z_{1} Z_{2}}{Z_{1}+Z_{2}} A_{I}\left(u_{2}-u_{1}\right), \\
\frac{\partial\left(h_{2} \rho_{2} u_{2}\right)}{\partial t}+\frac{\partial\left(h_{2} \rho_{2} u_{2}^{2}+h_{2} p_{2}\left(\rho_{2}\right)+\frac{1}{2} \rho_{2} g h_{2}^{2}\right)}{\partial x}=-\rho_{2} g h_{2} \frac{\partial h_{1}}{\partial x}+p_{0} \frac{\partial h_{2}}{\partial x}-\frac{Z_{1} Z_{2}}{Z_{1}+Z_{2}} A_{I}\left(u_{2}-u_{1}\right) .
\end{array}\right.
$$

$Z_{k}=\rho_{k} c_{k}$ denotes the acoustic impedance of fluid $k$ and $A_{I}$ denotes the specific interfacial area. $\rho_{1}=3.506 \mathrm{~kg} \cdot \mathrm{m}^{-3}, c_{1}=218 \mathrm{~m} \cdot \mathrm{s}^{-1}$ for krypton and $\rho_{2}=1.29 \mathrm{~kg} \cdot \mathrm{m}^{-3}, c_{2}=340 \mathrm{~m} \cdot \mathrm{s}^{-1}$ for air are used in this work. This acoustic drag effect modeling was developed in Saurel et al. (2003) [6] and Chinnayya et al. (2004) [25], pages 504 and 510. It is obtained by local interfacial pressure integration over the surface of a piece of interface. The interfacial pressure is estimated through an approximate Riemann solver for the Euler equations of gas dynamics. Let us mention that upon integration over height, as done in Eqs. (5.1) and (5.2), the specific interfacial area $A_{I}$ becomes dimensionless.

As shown in Figs. 18 and 19, computed results are significantly improved when drag effects are considered. The two figures show the solutions with respectively $A_{I}=0.0003$ and $A_{I}=$ 0.00015. A comparison of the different results at time $t=8 \mathrm{~s}$ is presented in Fig. 20, showing the influence of the $A_{I}$ parameter.

Indeed, the overall solution presents very good agreement with the 2D simulation using the diffuse interface two-phase flow model. The results show the ability of the new two-layer shallow water model to predict the spreading and dispersal of two gases evolving at low Mach number. The numerical advantages of this system are significant. In addition to its simple resolution through the HLL-type Riemann solver (Section 3.2), computational time saving is tremendous. For instance, the computation of the preceding test problem required about 30 hours for the two-dimensional simulation (computing only half of the domain) with a parallel code (MPI) running on 64 cores, while the one-dimensional computation needed only a couple of seconds in sequential implementation. 

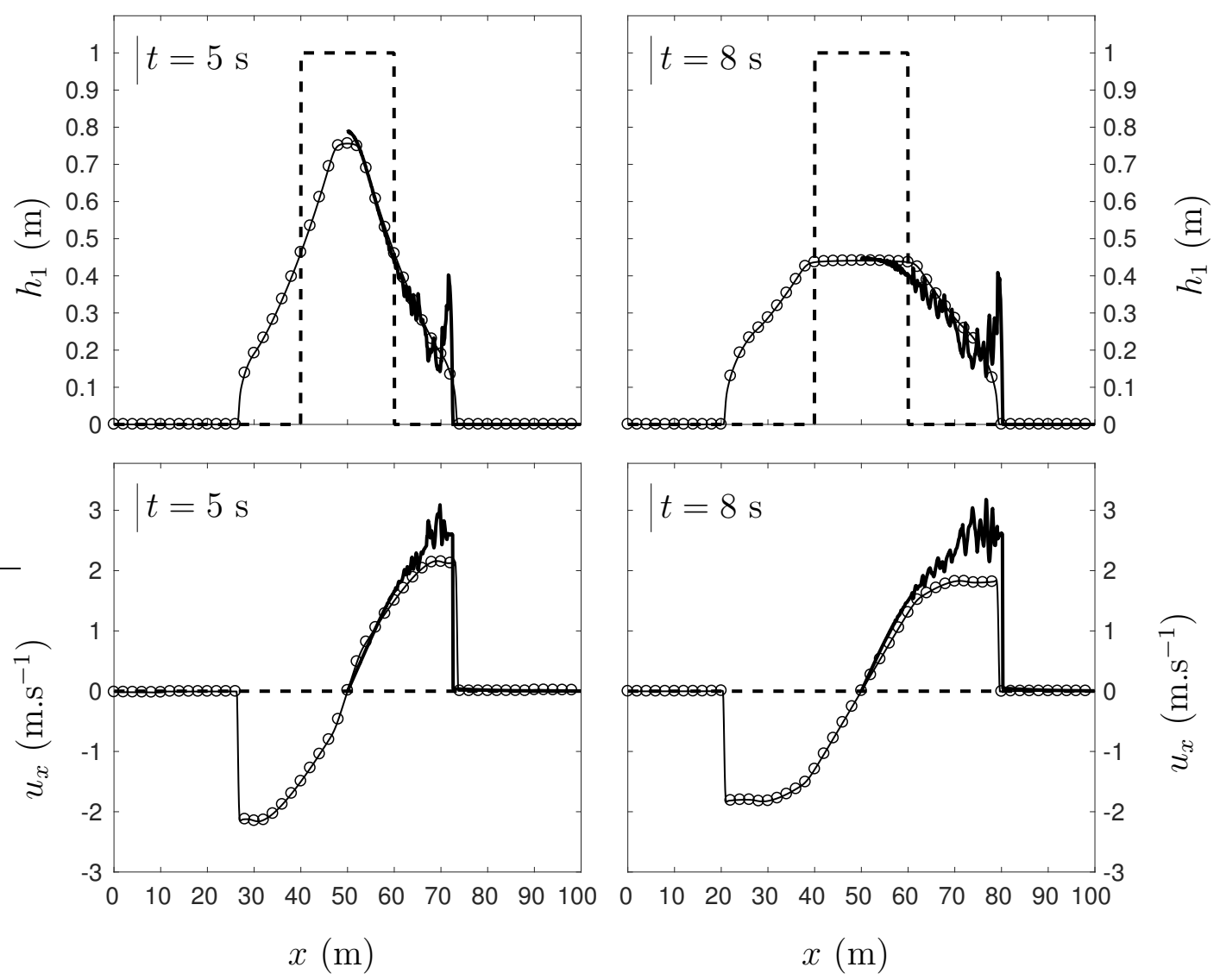

Figure 18: Comparison of the one-dimensional solution (thin lines and symbols) of the present two-layer shallow water model (HLL-type solver, $\theta_{k}=2$ ) versus the two-dimensional computation of the diffuse interface model of Saurel et al. (2009) 24] (thick lines). Drag effects are included in the shallow water system with $A_{I}=0.0003$. For the sake of clarity, 50 symbols are plotted for the two-layer solution. The column at left shows the results at time $t=5 \mathrm{~s}$ and the column at right shows the same results at time $t=8 \mathrm{~s}$. For symmetry reasons, only half of the numerical domain is computed with the two-dimensional simulation. Both computations (two-layer and diffuse interface systems) use $C F L=0.8$. 

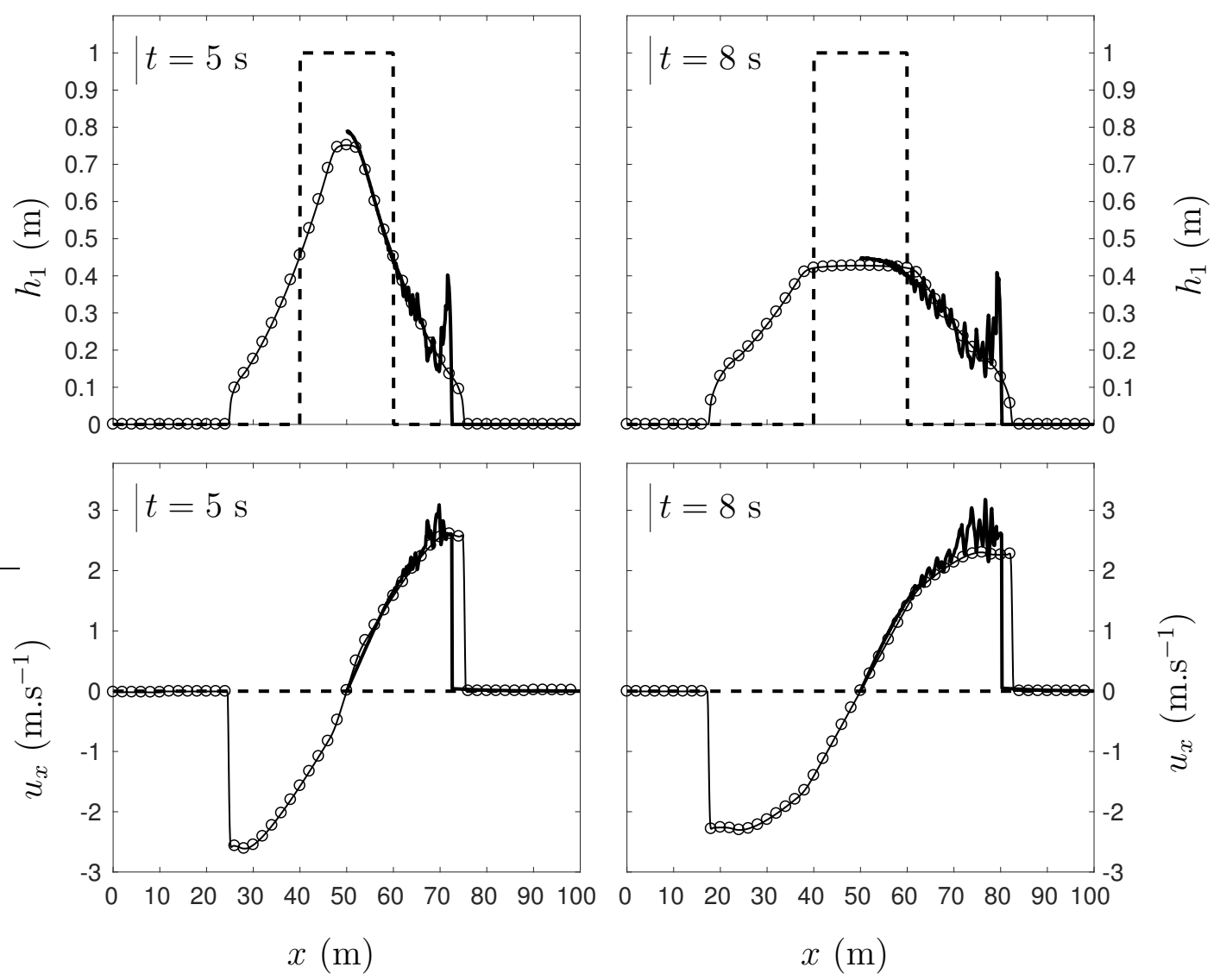

Figure 19: Comparison of the one-dimensional solution (thin lines and symbols) of the present two-layer shallow water model (HLL-type solver, $\theta_{k}=2$ ) versus the two-dimensional computation of the diffuse interface model of Saurel et al. (2009) 24] (thick lines). Drag effects are included in the shallow water system with $A_{I}=0.00015$. For the sake of clarity, 50 symbols are plotted for the two-layer solution. The column at left shows the results at time $t=5 \mathrm{~s}$ and the column at right shows the same results at time $t=8 \mathrm{~s}$. For symmetry reasons, only half of the numerical domain is computed with the two-dimensional simulation. Both computations (two-layer and diffuse interface systems) use $C F L=0.8$. 

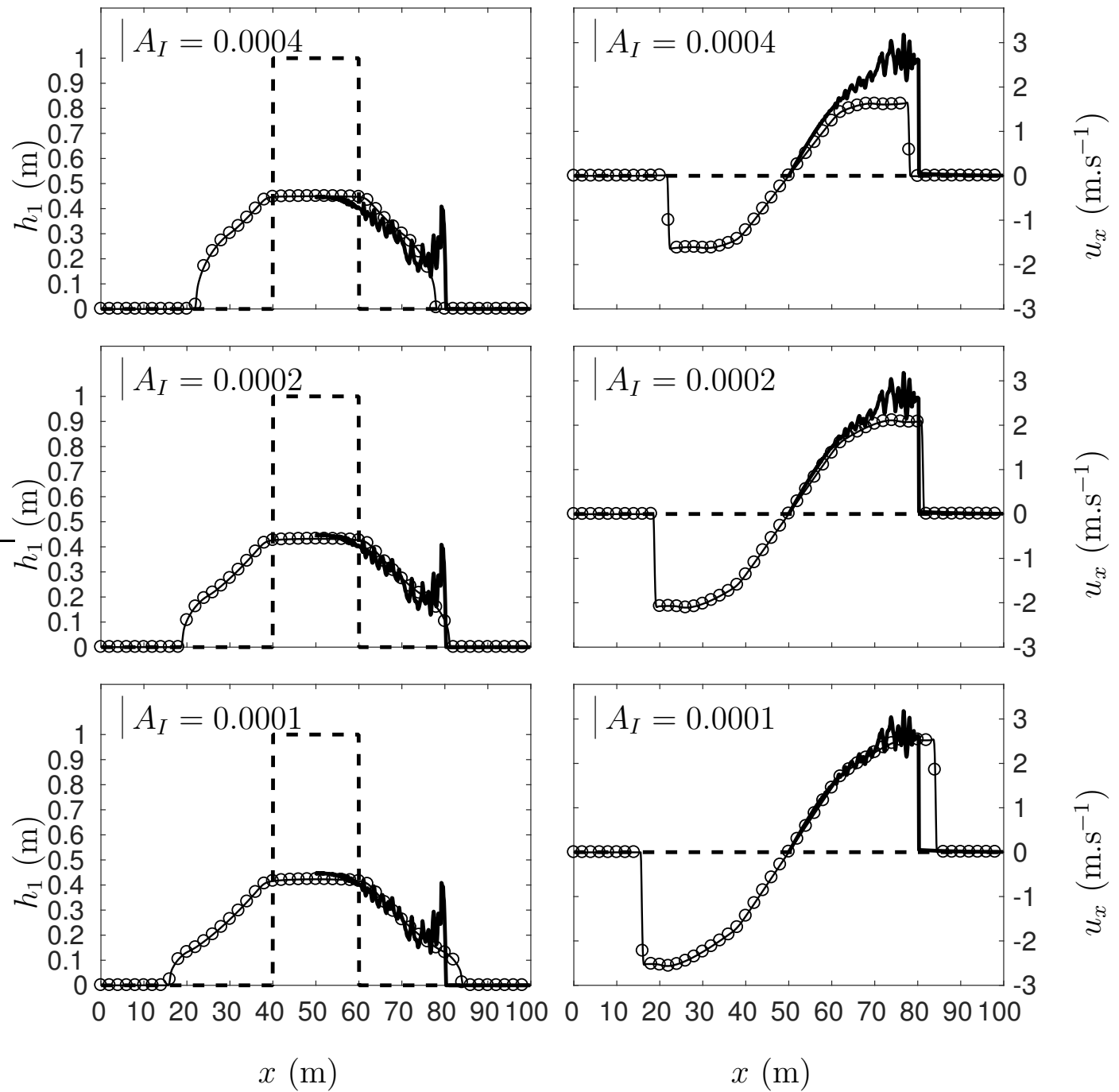

Figure 20: Influence of the interfacial area in drag force between the two layers. The solutions of the present onedimensional two-layer shallow water system (HLL-type solver, $\theta_{k}=2$ ) are displayed in thin lines and symbols. The solution of the two-dimensional computation of the diffuse interface model of Saurel et al. (2009) 24] is shown in thick lines. Drag effects are included in the shallow water system with various values of the specific interfacial area $A_{I}$. For the sake of clarity, 50 symbols are plotted for the two-layer solution. The results are shown at time $t=8 \mathrm{~s}$. For symmetry reasons, only half of the numerical domain is computed with the twodimensional simulation. Both computations (two-layer and diffuse interface systems) use $C F L=0.8$. $1 \mathrm{D}$ and $2 \mathrm{D}$ computations are in good agreement with $A_{I}=0.0002$. 


\section{Conclusion}

A pressure relaxation model with 6 equations has been built, especially devoted to twolayer shallow water flows. The mathematical structure of the new formulation is well-posed and results in a strictly hyperbolic model. The system considers weak compressibility of the fluids, which is responsible for its hyperbolic behavior, and is shown to tend to the conventional, but conditionally hyperbolic, two-layer shallow water model in the stiff pressure relaxation limit.

A simple, efficient and robust HLL-type Riemann solver has been derived to solve the corresponding non-conservative system. Computational examples have shown capabilities of the present formulation.

Compared to multi-D computations of gravity-driven interfacial flows, the new model offers tremendous numerical advantages and computational savings. This is done at the price of a single parameter in the drag force model.

This research work can be continued in many directions. Among them, the consideration of variable topography, friction with the ground and interfacial area creation through turbulence modeling seem important.

\section{Acknowledgements}

The authors are very grateful to Jeaniffer Vides for participating in multiple valuable discussions that definitely helped to improve the quality of this work.

Part of this work has been carried out in the framework of the Labex MEC (ANR-10-LABX0092) and of the A*MIDEX project (ANR-11-IDEX-0001-02) funded by ANR.

Support from CEA Gramat and especially Emmanuel Labedie are particularly acknowledged. The authors are grateful to the referees for their interesting and constructive comments. 


\section{References}

[1] F. Marble, Dynamics of a gas containing small solid particles, Combustion and Propulsion (5th AGARD Colloquium) (1963) 175-213.

[2] M. Baer, J. Nunziato, A two-phase mixture theory for the deflagration-to-detonation transition (DDT) in reactive granular materials, International Journal of Multiphase Flow 12 (6) (1986) 861-889.

[3] R. Saurel, A. Chinnayya, Q. Carmouze, Modelling compressible dense and dilute two-phase flows, Physics of Fluids 29 (6) (2017) 063301.

[4] M. Lallemand, R. Saurel, Pressure relaxation procedures for multiphase compressible flows, Tech. rep., INRIA (2000).

[5] A. Forestier, J. Hérard, X. Louis, Solveur de type Godunov pour simuler les écoulements turbulents compressibles, Comptes Rendus de l'Académie des Sciences. Paris Série 1, Mathématique 324 (8) (1997) 919-926.

[6] R. Saurel, S. Gavrilyuk, F. Renaud, A multiphase model with internal degrees of freedom: Application to shock-bubble interaction, Journal of Fluid Mechanics 495 (2003) 283-321.

[7] D. Lhuillier, C. Chang, T. Theofanous, On the quest for a hyperbolic effective-field model of disperse flows, Journal of Fluid Mechanics 731 (2013) 184-194.

[8] G. Richard, S. Gavrilyuk, A new model of roll waves: comparison with Brock's experiments, Journal of Fluid Mechanics 698 (2012) 374-405.

[9] S. Gavrilyuk, V. Liapidevskii, A. Chesnokov, Spilling breakers in shallow water: Applications to Favre waves and to the shoaling and breaking of solitary waves, Journal of Fluid Mechanics 808 (2016) 441-468.

[10] R. Abgrall, S. Karni, Two-layer shallow water system: A relaxation approach, SIAM Journal on Scientific Computing 31 (3) (2009) 1603-1627. 
[11] T. Gallouet, J. Masella, Un schéma de Godunov approché, Comptes Rendus de l'Académie des Sciences. Paris Série 1, Mathématique 323 (1) (1996) 77-84.

[12] L. Ovsyannikov, Two-layer shallow water model, Journal of Applied Mechanics and Technical Physics 20 (2) (1979) 127-135.

[13] A. Kurganov, G. Petrova, Central-upwind schemes for two-layer shallow water equations, SIAM Journal on Scientific Computing 31 (3) (2009) 1742-1773.

[14] R. Monjarret, Local well-posedness of the two-layer shallow water model with free surface, SIAM Journal on Applied Mathematics 75 (5) (2015) 2311-2332.

[15] A. Kapila, R. Menikoff, J. Bdzil, S. Son, D. Stewart, Two-phase modeling of deflagrationto-detonation transition in granular materials: Reduced equations, Physics of Fluids 13 (10) (2001) 3002-3024.

[16] R. Saurel, R. Abgrall, A multiphase Godunov method for compressible multifluid and multiphase flows, Journal of Computational Physics 150 (2) (1999) 425-467.

[17] A. Harten, P. Lax, B. Leer, On Upstream Differencing and Godunov-Type Schemes for Hyperbolic Conservation Laws, SIAM Review 25 (1) (1983) 35-61.

[18] R. LeVeque, Finite volume methods for hyperbolic problems, Vol. 31, Cambridge University Press, 2002.

[19] E. Toro, Riemann solvers and numerical methods for fluid dynamics: A practical introduction, Springer Science \& Business Media, 2013.

[20] S. Davis, Simplified second-order Godunov-type methods, SIAM Journal on Scientific and Statistical Computing 9 (3) (1988) 445-473.

[21] A. Chiapolino, R. Saurel, B. Nkonga, Sharpening diffuse interfaces with compressible fluids on unstructured meshes, Journal of Computational Physics 340 (2017) 389-417. 
[22] B. van Leer, Towards the ultimate conservative difference scheme. II. Monotonicity and conservation combined in a second-order scheme, Journal of Computational Physics 14 (4) (1974) 361-370.

[23] P. Sweby, High resolution schemes using flux limiters for hyperbolic conservation laws, SIAM Journal on Numerical Analysis 21 (5) (1984) 995-1011.

[24] R. Saurel, F. Petitpas, R. Berry, Simple and efficient relaxation methods for interfaces separating compressible fluids, cavitating flows and shocks in multiphase mixtures, Journal of Computational Physics 228 (5) (2009) 1678-1712.

[25] A. Chinnayya, E. Daniel, R. Saurel, Modelling detonation waves in heterogeneous energetic materials, Journal of Computational Physics 196 (2) (2004) 490-538.

\section{AppendixA. Extended formulation with variable external pressure}

To account for variable external pressure, System (2.3) is generalized as,

$$
\left\{\begin{array}{l}
\frac{\partial h_{1}}{\partial t}+u_{1} \frac{\partial h_{1}}{\partial x}=\frac{\mu\left(p_{1}-p_{0}-\rho_{2} g h_{2}\right)}{\rho_{1} c_{1}^{2}} \\
\frac{\partial\left(h_{1} \rho_{1}\right)}{\partial t}+\frac{\partial\left(h_{1} \rho_{1} u_{1}\right)}{\partial x}=0 \\
\frac{\partial\left(h_{1} \rho_{1} u_{1}\right)}{\partial t}+\frac{\partial\left(h_{1} \rho_{1} u_{1}^{2}+h_{1} p_{1}\left(\rho_{1}, \rho_{2}, h_{2}\right)+\frac{1}{2} \rho_{1} g h_{1}^{2}\right)}{\partial x}=\rho_{2} g h_{2} \frac{\partial h_{1}}{\partial x}+p_{0} \frac{\partial h_{1}}{\partial x} \\
\frac{\partial h_{2}}{\partial t}+u_{2} \frac{\partial h_{2}}{\partial x}=\frac{\mu\left(p_{2}-p_{0}\right)}{\rho_{2} c_{2}^{2}}, \\
\frac{\partial\left(h_{2} \rho_{2}\right)}{\partial t}+\frac{\partial\left(h_{2} \rho_{2} u_{2}\right)}{\partial x}=0, \\
\frac{\partial\left(h_{2} \rho_{2} u_{2}\right)}{\partial t}+\frac{\partial\left(h_{2} \rho_{2} u_{2}^{2}+h_{2} p_{2}\left(\rho_{2}\right)+\frac{1}{2} \rho_{2} g h_{2}^{2}\right)}{\partial x}=-\rho_{2} g h_{2} \frac{\partial h_{1}}{\partial x}+p_{0} \frac{\partial h_{2}}{\partial x}
\end{array}\right.
$$

With this formulation, the equation of state of the first (heaviest) fluid transforms to,

$$
p_{1}=p_{0}+\rho_{2} g h_{2}+c_{1}^{2}\left(\rho_{1}-\rho_{1}^{(0)}\right)
$$

while it is unchanged for the upper layer,

$$
p_{2}=p_{0}+c_{2}^{2}\left(\rho_{2}-\rho_{2}^{(0)}\right)
$$


The $g \rho_{2} h_{1} h_{2}$ flux term present in System (2.3) is now considered in the equation of state of the first fluid. The hydrostatic pressure of the first fluid has been consequently reformulated in the relaxation term of the height equation as well. It is important to check that the hyperbolicity of the reformulated system is still valid. Also, the stiff pressure relaxation limit is meant to recover Ovsyannikov's [12] system (Eq. (2.1) ). These points are examined hereafter.

System (A.1) is expressed in primitive-variable formulation (without source terms) as,

$$
\frac{\partial W}{\partial t}+A(W) \frac{\partial W}{\partial x}=0
$$

with

$$
W=\left(\begin{array}{l}
h_{1} \\
h_{2} \\
\rho_{1} \\
\rho_{2} \\
u_{1} \\
u_{2}
\end{array}\right), \quad A(W)=\left(\begin{array}{cccccc}
u_{1} & 0 & 0 & 0 & 0 & 0 \\
0 & u_{2} & 0 & 0 & 0 & 0 \\
0 & 0 & u_{1} & 0 & \rho_{1} & 0 \\
0 & 0 & 0 & u_{2} & 0 & \rho_{2} \\
\frac{p_{1}-p_{0}-\rho_{2} g h_{2}}{h_{1} \rho_{1}}+g & 0 & \frac{c_{1}^{2}+\frac{1}{2} g h_{1}}{\rho_{1}} & 0 & u_{1} & 0 \\
g & \frac{p_{2}-p_{0}}{h_{2} \rho_{2}}+g & 0 & \frac{c_{2}^{2}+\frac{1}{2} g h_{2}}{\rho_{2}} & 0 & u_{2}
\end{array}\right) .
$$

The wave speeds are solutions of $\operatorname{det}(A(W)-\lambda \underline{\underline{I}})=0$ resulting in,

$$
\left(u_{1}-\lambda\right)\left(u_{2}-\lambda\right)\left[\left(u_{2}-\lambda\right)^{2}-\left(c_{2}^{2}+\frac{1}{2} g h_{2}\right)\right]\left[\left(u_{1}-\lambda\right)^{2}-\left(c_{1}^{2}+\frac{1}{2} g h_{1}\right)\right]=0
$$

The six real and distinct eigenvalues of System (2.3) are recovered,

$$
\left\{\begin{array}{lll}
\lambda_{1}=u_{1}, & \lambda_{2}=u_{1}+\sqrt{c_{1}^{2}+\frac{1}{2} g h_{1}}, & \lambda_{3}=u_{1}-\sqrt{c_{1}^{2}+\frac{1}{2} g h_{1}}, \\
\lambda_{4}=u_{2}, & \lambda_{5}=u_{2}+\sqrt{c_{2}^{2}+\frac{1}{2} g h_{2}}, & \lambda_{6}=u_{2}-\sqrt{c_{2}^{2}+\frac{1}{2} g h_{2} .}
\end{array}\right.
$$

System (A.1), like System (2.3), is therefore strictly hyperbolic. 


\section{Asymptotic limit}

Let us now consider the following expansion,

$$
\left\{\begin{array}{l}
p_{1}=p_{1}^{(0)}+\epsilon p_{1}^{(1)}+\ldots, \\
p_{0}+h_{2} \rho_{2} g=\left(p_{0}+h_{2} \rho_{2} g\right)^{(0)}+\epsilon\left(p_{0}+h_{2} \rho_{2} g\right)^{(1)}+\ldots,
\end{array}\right.
$$

with $\epsilon=\frac{1}{\mu}$. The first equation of System (A.1) becomes,

$$
\frac{\partial h_{1}}{\partial t}+u_{1} \frac{\partial h_{1}}{\partial x}=\frac{1}{\epsilon} \frac{p_{1}^{(0)}-\left(p_{0}+\rho_{2} g h_{2}\right)^{(0)}}{\rho_{1} c_{1}^{2}}+\frac{p_{1}^{(1)}-\left(p_{0}+\rho_{2} g h_{2}\right)^{(1)}}{\rho_{1} c_{1}^{2}} .
$$

Hence, at leading order,

$$
p_{1}^{(0)}=\left(p_{0}+h_{2} \rho_{2} g\right)^{(0)} .
$$

This last result is introduced in the momentum equation of the first fluid of System (A.1) and yields,

$$
\frac{\partial\left(h_{1} \rho_{1} u_{1}\right)}{\partial t}+\frac{\partial\left(h_{1} \rho_{1} u_{1}^{2}+\frac{1}{2} \rho_{1} g h_{1}^{2}+g \rho_{2} h_{1} h_{2}\right)}{\partial x}=\rho_{2} g h_{2} \frac{\partial h_{1}}{\partial x} .
$$

The momentum equation of System (2.1) is thus recovered. Similar manipulations on the second layer momentum equation of the present model lead to,

$$
\frac{\partial\left(h_{2} \rho_{2} u_{2}\right)}{\partial t}+\frac{\partial\left(h_{2} \rho_{2} u_{2}^{2}+\frac{1}{2} \rho_{2} g h_{2}^{2}\right)}{\partial x}=-\rho_{2} g h_{2} \frac{\partial h_{1}}{\partial x} .
$$

The second momentum equation of System (2.1) is recovered as well. The present formulation thus tends in the limit of stiff pressure relaxation to the conventional two-layer Saint-Venant model. Thereby, System (A.1) allows to compute solutions of the non-hyperbolic model [12] with a hyperbolic step using the preceding wave speeds (Eq. (A.7)) followed by a correction step regarding the heights.

This latter is supposed to be isentropic. The equation of state for the second fluid (lightest) reads: $p_{2}=p_{0}+c_{2}^{2}\left(\rho_{2}-\rho_{2}^{(0)}\right)$. At the end of the relaxation step, $p_{2}^{*}=p_{0}$ and implies $\rho_{2}^{*}=$ $\rho_{2}^{(0)}$. As the mass quantity is known from the hyperbolic evolution and is constant during the 
relaxation process, $m_{2}=h_{2} \rho_{2}=h_{2}^{*} \rho_{2}^{*}$, the relaxation step just consists in reseting the height: $h_{2}^{*}=\frac{h_{2} \rho_{2}}{\rho_{2}^{(0)}}$.

For the first fluid, the EOS now reads: $p_{1}=p_{0}+\rho_{2}^{*} g h_{2}^{*}+c_{1}^{2}\left(\rho_{1}-\rho_{1}^{(0)}\right)$. At the end of the relaxation process, the pressure reads $p_{1}^{*}=p_{0}+\rho_{2}^{*} g h_{2}^{*}$. Consequently, $\rho_{1}^{*}=\rho_{1}^{(0)}$ and the relaxation step reduces to $h_{1}^{*}=\frac{h_{1} \rho_{1}}{\rho_{1}^{(0)}}$ as before with System (2.3) . The only difference is that $\rho_{1}^{(0)}$ no longer represents the density of fluid 1 at atmospheric pressure $p_{0}$ but is corrected by hydrostatic effects as $\rho_{1}^{(0)}=\rho_{1}^{(00)}+\frac{\rho_{2}^{*} g h_{2}^{*}}{c_{1}^{2}}$, where $\rho_{1}^{(00)}$ represents the density of fluid 1 at atmospheric pressure.

System (A.1) is then an extended formulation of System (2.3). Formulation (A.1) provides another interpretation of the flux term $g \rho_{2} h_{1} h_{2}$ that is now expressed through the equation of state of the first layer (heaviest). Besides, this extended formulation seems more attractive for a multi-layer extension of the present model.

At the discrete level this model does not pose extra computational difficulties. It has been coded and tested on the example of Fig. 15 with exactly the same results as those of Fig. 20. 